\title{
PLANT COMMUNITIES WITH PINUS MUGO (ALLIANCE PINION MUGO) IN THE SUBALPINE BELT OF THE WESTERN CARPATHIANS - A NUMERICAL APPROACH
}

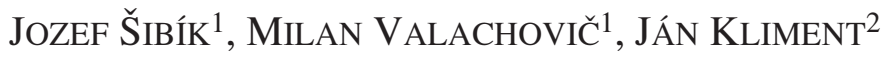 \\ ${ }^{1}$ Institute of Botany, Slovak Academy of Sciences \\ Dúbravská cesta 14, SK-845 23 Bratislava, Slovak Republic \\ e-mail: jozef.sibik@savba.sk \\ 2 Botanical Garden of Comenius University \\ SK-038 15 Blatnica, Slovak Republic
}

(Received: February 11, 2005. Accepted: June 30, 2005)

\begin{abstract}
A syntaxonomical revision of plant communities with dominant Pinus mugo in the Western Carpathians is presented. The data set of 341 relevés was examined and analysed using the detrended correspondence analysis and the cluster analysis. Major gradients and clusters were interpreted using Ellenberg's indicator values. The major gradient in species composition was associated with available nutrients and moisture.

The authors suggest distinguishing the dwarf pine stands of the supramontanous and subalpine belts of the Western Carpathians referred to the alliance Pinion mugo Pawłowski in Pawłowski et al. 1928 of the order JuniperoPinetalia mugo Boşcaiu 1971 and the class Roso pendulinae-Pinetea mugo Theurillat in Theurillat et al. 1995, into three separate associations: the Cetrario-Pinetum mugo Hadač 1956, the Homogyno alpinae-Pinetum mugo (Sillinger 1933) nom. nov., and the Adenostylo alliariae-Pinetum mugo (Sillinger 1933) Šoltésová 1974.

The authors also elucidated the unauthorized name of the association Vaccinio myrtilli-Pinetum mugo Hadač 1956, which is a younger homonym of the valid name of the association Vaccinio myrtilli-Pinetum montanae Morton 1927 that characterises the acidophilous dwarf pine stands on calcareous bedrocks in the Alps.
\end{abstract}

KEY WORDS: Pinus mugo, dwarf mountain pine stands, numerical classification, ordination, syntaxonomy.

\section{INTRODUCTION}

The first geobotanical works on the distribution of dwarf pine stands in the subalpine belt of the Western Carpathians pertain to the Polish authors Szafer, Pawłowski and Kulczyński (1923). They studied plant communities in the Chochołowska Dolina valley in the Western Tatra Mts, where they described the community Pinetum montanae fruticosae. Proceeding with field works few years later (Szafer et al. 1927), they described the association Pinetum mughi (=Mughetum) carpaticum from the Dolina Kościeliska valley in the Western Tatra Mts. The authors distinguished a floristically richer community $\mathrm{Mu}-$ ghetum calcicolum on calcareous soils and a floristically poorer community Mughetum silicicolum on acid soils with dominant Vaccinium myrtillus in underbrush. Similar appreciation was later made by Pawłowski (1928) and Pawłowski, Sokołowski and Wallisch (1928) following a survey in a valley surrounding the Morskie Oko Lake.
They included the Pinus mugo scrubs to the separate alliance, the Pinion mughi.

In Krajina (1933) the interpretation is based on a more floristic approach, while in Hadač (1956) principally on structure of dwarf pine scrubs. Generally, only the work of Šoltésová (1974) may be regarded as the first complete publication containing and comparing the vegetation data on Pinus mugo scrubs from all the Western Carpathians area. She came out from the first Sillinger's conception (Sillinger 1933) and divided the phytocoenoses into two associations: the Vaccinio myrtilli-Pinetum mughi (Sillinger 1933) Šoltésová 1974 and the Adenostylo alliariae-Pinetum mughi (Sillinger 1933) Šoltésová 1974. She integrated both associations to the suballiance Pinion mughi and the alliance Vaccinio-Piceion. Hadač (1985) in the List of Vegetation Units of Slovakia (Mucina and Maglocký 1985) made a revision of a broad number of names and opinions on the classification of this type of vegetation. He combined his own view on this problem with that of Šoltésová 
(cf. Šoltésová 1974). This enabled him to distinguish three associations: the Adenostylo-Pinetum mugo (Sillinger 1933) Šoltésová 1974, the Athyrio distentifolii-Pinetum mugo (Hadač 1956) Hadač in Mucina et Maglocký 1985, and the Vaccinio myrtilli-Pinetum mugo Hadač 1956 incorporating them to the alliance Pinion mugo and the class Vaccinio-Piceetea (see also Fig. 1).

Although the Pinus mugo scrubs look like a homogeneous community, the large discrepancies in their classification are apparent not only in the Western Carpathian Mts but also in the Alps and mountains in the Balkan Peninsula (cf.: Aichinger 1933; Braun-Blanquet et al. 1939; Wallnöfer 1993a, b; Roussakova 2000; Poldini et al. 2004). The possibility to compare classical phytocoenological methods with the numerical approach led the authors to this study. The folloving goals were set: a) to find out whether the classical vegetation types which were set in the past using classical and much-less deductive phytocenological methods can be sustainable nowadays; b) to decide whether the discrepancy between the particular vegetation types is important enough to be classified in different higher syntaxa (alliances and/or classes); c) to find out which ecological factor has the director influence on the variability of the dwarf pine shrubs. Last but not least, we tried to emphasize the appropriate classification of these specific looking coppices into a separate class.

\section{MATERIAL AND METHODS}

The syntaxonomical revision included 341 phytocoenological relevés of plant communities with the dominance and/or high abundance of Pinus mugo in the supramontanous and subalpine belts of the central part of the Western Carpathians. The examination was carried out from the data gained in the time span from 1923-2003.

Phytocoenological relevés of plant communities from ombrotrophic and oligotrophic raised bogs, broadly classified to the class Oxycocco-Sphagnetea (cf. Šoltés et al. 2001), were excluded.

All relevés have been collected according to the principles of Zürich-Montpelliér school (Braun-Blanquet 1964). Different scales of abundance and dominance have been used by various authors (Table 1). To obtain the data comparable within the numerical classification, all relevés were transformed into the nine-degree ordinal scale (van den Maarel 1979). The taxa determined only at the level of genus were excluded (except the genus Alchemilla, Polytrichum and Sphagnum). Some taxa were classified within the higher or more broadly defined taxa: Aconitum firmum (subsp. firmum, subsp. moravicum), Alchemilla sp. div. (A. crinita, A. glabra, A. glaucescens, A. incisa, A. monticola, A. xanthochlora), Anthoxanthum odoratum agg. (A. alpinum), Cardamine amara (subsp. opicii), Cardaminopsis arenosa agg. (C. borbasii), Cladonia gracilis [var. aspera Flörke, var. elongata (Jacq.) Fr.], Cladonia ciliata (var. tenuis), Dactylis glomerata (subsp. slovenica), Dryopteris dilatata s. 1. (D. carthusiana), Empetrum nigrum s. 1. (E. hermaphroditum), Galeobdolon luteum s. 1. (G. montanum), Helianthemum grandiflorum (subsp. glabrum, subsp. grandiflorum, subsp. obscurum), Hieracium alpinum agg. (H. halleri, H. pseudofritzei Benz et Zahn), Jovibarba globifera (subsp. glabrescens, subsp. tatrensis), Lescuraea mutabilis (var. saxicola Hagen), Leucanthemum vulgare agg. (L. margaritae), Lotus corniculatus (var. alpicola Beck), Luzula luzuloides (subsp. luzuloides, subsp. rubella), Myosotis palustris agg. (M. nemorosa), Pimpinella major (subsp. rhodochlamys), Polytrichum sp. div. (P. alpinum, P. commune, $P$. formosum), Senecio nemorensis agg. (S. germanicus, S. hercynicus, S. ovatus), Sorbus aucuparia (subsp. glabrata), Sphagnum sp. div. (S. capillifolium, S. fallax, S. girgensohnii, S. magellanicum, S. quinquefarium, S. russowii), Solidago virgaurea (subsp. minuta), Swertia perennis (subsp. alpestris), Soldanella hungarica (subsp. major), Sempervivum wettsteinii (subsp. wettsteinii), Thymus pulcherrimus (subsp. sudeticus). Numerical classification was performed by the program NCLAS from the SYN-TAX 5 package (Podani 1993). The $\beta$-flexible method $(\beta=-0.25)$ with Euclidean distance and Jaccard's, Ružička's and Wishart's similarity coefficients were used. As phytocoenological data consisted of large data records covering high proportion of actual variability of dwarf pine stands, about 40 relevés have hit different clusters applying different coefficients. These were classified again, being evaluated by comparison and over phytocoenological tables.

Major gradients in species composition of the Pinus mugo scrubs were analysed through ordination of this data set, using the detrended correspondence analysis (DCA) from the CANOCO 4.5 package (ter Braak and Šmilauer 2002) suitable for the analysis of heterogeneous data. For ecological interpretation of the ordination axes, average Ellenberg indicator values (Ellenberg et al. 1992) for relevés were plotted onto DCA ordination diagram as supplementary environmental data.

The contents of each column in Table 2 comprise the number of relevés used for the synthesis, and the average number of species in the relevant community. Each taxon is characterised by the frequency ("Freq" in Table; in \%; $+=$ frequency $<0.5 \%$ ), by the fidelity ("Fide" in Table; in phi coefficient multiplied by 100) and the mean value of abundance (upper index) calculated over the FYTOPACK (Jarolímek and Schlosser 1997). The individual columns contain also brief references (for unpublished data only the names of authors are given), the number of relevés and their position in the level of orographical units according to the map from the Database of Fauna of Slovakia, scale 1:500 000. Diagnostically important taxa of individual plant communities were determined a posteriori, by calculating frequency and fidelity of each species to each cluster, using the phi coefficient of association in the program Juice 6.1 (Tichý 2002). These taxa are given in bold. The threshold phi value and frequency for a species to be considered as diagnostic was determined by combination of these, because the number of relevés in particular clusters was very variable.

The nomenclature of the taxa generally follows the Checklist of non-Vascular and Vascular Plants of Slovakia (Marhold and Hindák 1998). Only in few exceptions, the author's name is included. The subspecies (given without the species modifier) in the text, in Table 2, or in Fig. 2 are marked with asterisks $(*)$. The names of the syntaxa are according to Mucina and Maglocký (1985), except few ones which include author's name; the names of diagnostic taxa of higher syntaxa follow synthetic studies from Slovakia (Šoltés 1976; Jarolímek et al. 2002; Šibík et al. 2004; Kliment et al. 2004; Dúbravcová et al. 2005). 


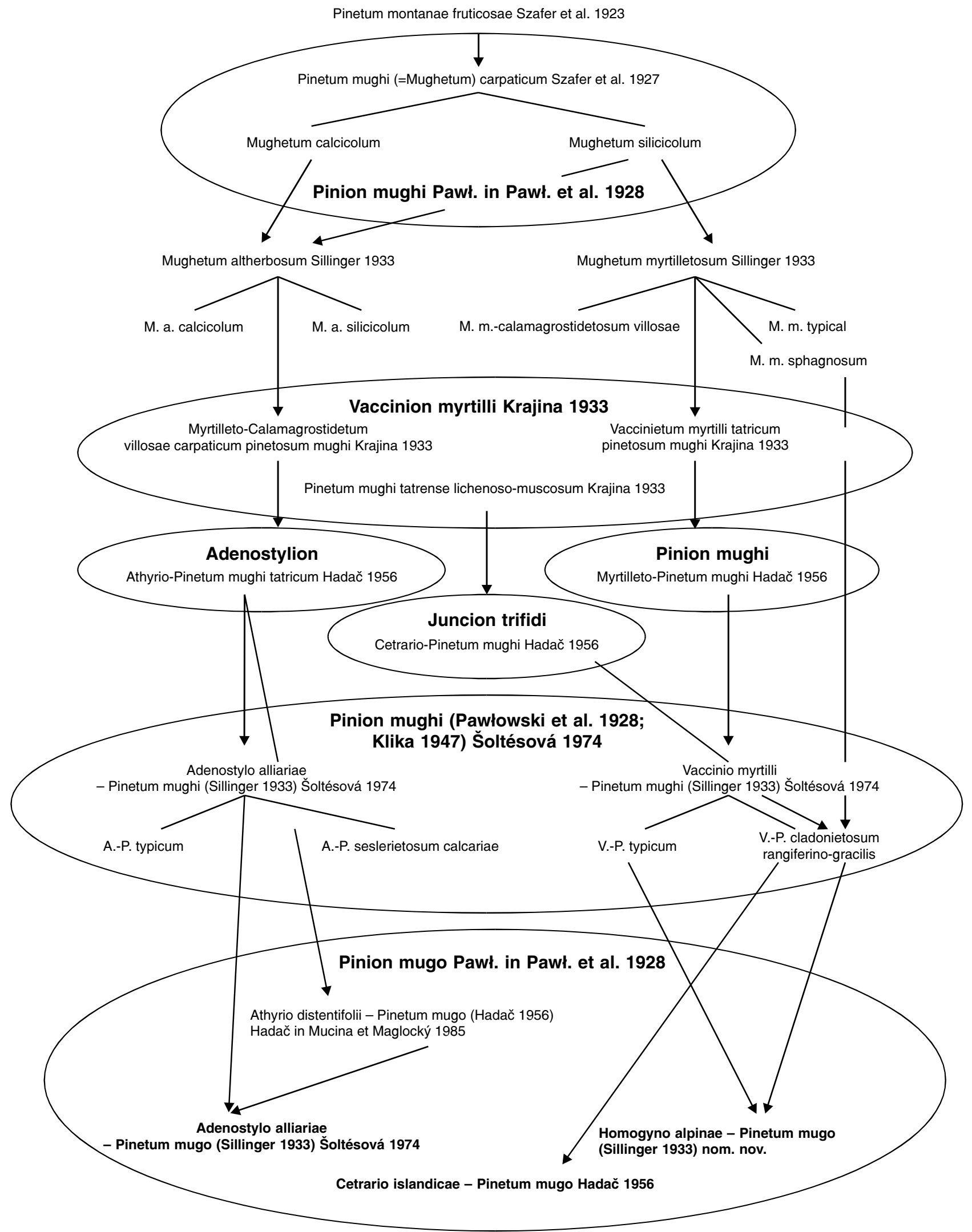

Fig. 1. Changes in the names of syntaxa in the Western Carpathians.

In the communities' descriptions, the following abbreviations are used: art. $=$ article as to the Code of Phytocoenological Nomenclature (Weber et al. 2000), const. = constantly companion taxon (frequency higher than $60 \%$ ), dif. $=$ differential taxon, dom. $=$ dominating taxon.
Study area

The study area was determined by the distribution of dwarf pine stands in the supramontanous and subalpine belts of the Krivánska Malá Fatra Mts, Lúčanská Malá Fatra Mts, Velká Fatra Mts, Chočské vrchy Mts, Nízke Tatry 
TABLE 1. Transformation of the different scales of abundance and dominance.

A - Braun-Blanquet 1964; B - Barkman et al. 1964; C - Hadač et al. 1969; D - Randuška et al. 1986; E - van den Maarel 1979.

\begin{tabular}{ccccc}
\hline A & B & C & D & E \\
\hline r & r & + & - & 1 \\
+ & + & 1,2 & + & 2 \\
1 & 1 & 3 & 1 & 3 \\
& $2 \mathrm{~m}$ & 4 & - & 4 \\
2 & $2 \mathrm{a}$ & 5 & -2 & 5 \\
& $2 \mathrm{~b}$ & 6 & +2 & 6 \\
3 & 3 & 7 & $-3,+3$ & 7 \\
4 & 4 & 8 & $-4,+4$ & 8 \\
5 & 5 & 9,10 & $-5,+5$ & 9 \\
\hline
\end{tabular}

Mts, Západné Tatry Mts, Vysoké Tatry Mts, Belianske Tatry Mts and Oravské Beskydy Mts - the massif of Babia hora Mt., and Pilsko Mt. (Fig. 3).

These regions belong to a cold climate. Mean monthly temperatures for January vary from -6 to $-9^{\circ} \mathrm{C}$, for July from 10 to $12^{\circ} \mathrm{C}$ and the average year temperature is $4^{\circ} \mathrm{C}$. Monthly precipitations are very changeable. Mean annual precipitation is from 1200 to $1400 \mathrm{~mm}$ (Lupin et al. 2002).

\section{RESULTS}

\section{Numerical classification}

In spite of using different coefficients of similarity for the numerical classification, gain results were almost identical. Interpreting them, we have applied the second level of division. The individual clusters on the dendrogram pertained to concrete, floristically well-defined communities (Table 2, Fig. 4).

Using the Jaccard's and Ružička's coefficients of similarity, the group of the relevés which represented most oligotrophic stands with high abundance of Sphagnum species, was attached to the cluster A (Fig. 4), however, the diagnostic taxa of the classes Caricetea curvulae Br.-Bl. 1948 and Loiseleurio-Vaccinietea Eggler ex Schubert 1960, which seemed to be differential for the association Cetrario-Pinetum mugo, were missing. The application of the Euclidean distance and the Wishart's coefficient of similarity brought nearly similar results, though the differences between individual clusters pointed out the disparities between individual communities. The relevés from individual table columns (Table 2) corresponded well with the dendrogram elaborated using the Wishart's coefficient of similarity (Fig. 4), however, the problematic relevés were re-shuffled.

\section{Cluster A:}

This group of relevés represented the association Cetrario islandicae-Pinetum mugo. This is a three-layered, species-poor community, where apart from the extremely dwarf Pinus mugo scrubs (a few decimeters in height), grasses (Festuca supina, Oreochloa disticha, Juncus trifidus) and dwarf shrubs (Vaccinium myrtillus, V. vitis-idaea) are distributed markedly sticking out. In the layer of bryophytes and lichens Cetraria islandica, Pleurozium schreberi and Polytrichum strictum with numerous Cladonia species dominate.
This phytocoenose occurs on extremely severe sites, in the interface of the subalpine and alpine belts, occupying windswept stands. This is an autochthonous community concentrated in granite and mylonite-composed parts of the Západné Tatry Mts and Vysoké Tatry Mts.

\section{Cluster B:}

This group represents the very poor in species and floristically monotonous community Homogyno alpinae-Pinetum mugo, of which the habitus define the dominant 1.5-3 $\mathrm{m}$ tall Pinus mugo scrubs. From other woody species, Salix silesiaca, Sorbus aucuparia, Picea abies and some other form a shrub layer. The spruce often develops typical flagged or tabled forms. A tree layer occurs rarely, with limited cover, primarily being formed by Sorbus aucuparia and $P i$ cea abies. The dominant bilberry, Vaccinium myrtillus, with several other species, such as Avenella flexuosa, Homogyne alpina and Calamagrostis villosa determine the physiognomy of the herb layer. The moss layer is usually well developed; with most frequently present Dicranum scoparium and Hylocomium splendens.

Associations Homogyno alpinae-Pinetum mugo are commonly concentrated in the supramontanous and subalpine belts of the central part of the Western Carpathians, spreading on the slopes with different inclination and orientation, mainly on siliceous bedrocks. The extreme climatic and edaphic conditions induce the occurrence of moderately acid-oligotrophic species in the herb and moss layers. This is an autochthonous community on siliceous bedrocks in the subalpine belt with the signs of climax vegetation. On calcareous substrata, only stands from the sites with a thick layer of undecomposed humus, which isolates plants from the bedrock, can be assigned to this community.

\section{Cluster C:}

This cluster represents a more-layered, species rich and floriferous shrubby community Adenostylo-Pinetum mugo, of which the physiognomy is determined by Pinus mugo. The tree layer, more commonly formed by Sorbus aucuparia, develops rarely and with very limited cover. The herb-layer is composed of the tall-herb taxa (Aconitum firmum, Adenostyles alliariae, Cicerbita alpina, Doronicum austriacum, Luzula sylvatica, Rubus idaeus, Veratrum *lobelianum, etc.), forming a more or less continuous layer. The medium or low herb layer consists of Festuca carpatica, Geranium sylvaticum, Hypericum maculatum, Leucanthemum rotundifolium, Polygonatum verticillatum, Primula elatior, Valeriana tripteris and some other species. Mosses are occurring relatively frequently, the most common species is Dicranum scoparium.

The community is well developed on the sites with favourable edaphic conditions (with sufficient nutrition supply during all the vegetation period) and with good decomposition of humus. The stands occupy relatively steep slopes harbouring calcareous and siliceous bedrocks. The rainfalls provide a constant reserve of soaked water all over the vegetation season, however, the layer of nutrient-productive soil with moderately acid reaction may be regarded as decisive for the community occurrence (Šoltésová 1974). The community occurs in the supramontanous and subalpine belts of all studied regions, except the Lúčanská Malá Fatra Mts. 
TABLE 2. Plant communities of the alliance Pinion mugo in the Western Carpathians (a brief synoptic table, species with frequency $>10 \%$ ). A - Cetrario islandicae - Pinetum mugo; B - Homogyno alpinae - Pinetum mugo; C - Adenostylo alliariae - Pinetum mugo

\begin{tabular}{|c|c|c|c|c|c|c|c|}
\hline \multicolumn{2}{|c|}{ Community } & \multicolumn{2}{|c|}{$\mathbf{A}$} & \multicolumn{2}{|c|}{$\mathbf{B}$} & \multicolumn{2}{|c|}{$\mathbf{C}$} \\
\hline \multicolumn{2}{|c|}{ Number of relevés } & \multicolumn{2}{|c|}{28} & \multicolumn{2}{|c|}{205} & \multicolumn{2}{|c|}{108} \\
\hline \multicolumn{2}{|c|}{ Average species number } & \multicolumn{2}{|c|}{20} & \multicolumn{2}{|c|}{16} & \multicolumn{2}{|c|}{34} \\
\hline & & $\begin{array}{c}\text { Freq } \\
(\%)\end{array}$ & $\begin{array}{l}\text { Fide } \\
\text { (phi) }\end{array}$ & $\begin{array}{c}\text { Freq } \\
(\%)\end{array}$ & $\begin{array}{l}\text { Fide } \\
\text { (phi) }\end{array}$ & $\begin{array}{c}\text { Freq } \\
(\%)\end{array}$ & $\begin{array}{l}\text { Fide } \\
\text { (phi) }\end{array}$ \\
\hline \multicolumn{8}{|c|}{ Differential taxa of the associations } \\
\hline $\mathrm{CC}$ & $\begin{array}{l}\text { Cetraria islandica }\left(\mathrm{E}_{0}\right) \\
\text { Cladonia rangiferina }\left(\mathrm{E}_{0}\right)\end{array}$ & $\begin{array}{c}100^{6} \\
68^{3}\end{array}$ & $\begin{array}{l}\mathbf{5 7 . 3} \\
\mathbf{5 5 . 8}\end{array}$ & $\begin{array}{c}19^{3} \\
7^{3}\end{array}$ & $\begin{array}{l}- \\
-\end{array}$ & $\begin{array}{l}6^{2} \\
2^{2}\end{array}$ & $\begin{array}{l}- \\
-\end{array}$ \\
\hline $\mathrm{CC}$ & Cladonia gracilis $\left(\mathrm{E}_{0}\right)$ & $61^{2}$ & 58.5 & $4^{2}$ & - & $1^{1}$ & - \\
\hline $\mathrm{CC}$ & Juncus trifidus & $57^{2}$ & 67.2 & $1^{2}$ & - & • & - \\
\hline $\mathrm{CC}$ & Cladonia arbuscula $\left(\mathrm{E}_{0}\right)$ & $\mathbf{5 0}^{2}$ & 54.5 & $3^{2}$ & - & • & - \\
\hline $\mathrm{CC}$ & Oreochloa disticha & $46^{2}$ & 66.6 & - & - & - & - \\
\hline $\mathrm{CC}$ & Festuca supina & $43^{2}$ & 63.9 & - & - & - & - \\
\hline $\mathrm{CC}$ & Pulsatilla scherfelii & $36^{3}$ & 49.8 & $1^{1}$ & - & $1^{2}$ & - \\
\hline & Alectoria ochroleuca $\left(\mathrm{E}_{0}\right)$ & $32^{2}$ & 51.8 & $+^{3}$ & - & - & - \\
\hline & Cladonia squamosa $\left(\mathrm{E}_{0}\right)$ & $32^{2}$ & 44.2 & $2^{3}$ & - & - & - \\
\hline $\mathrm{CC}$ & Campanula alpina & $32^{2}$ & 55.0 & - & - & - & - \\
\hline $\mathrm{CC}$ & Hieracium alpinum agg. & $29^{2}$ & 51.8 & - & - & - & - \\
\hline LV & Huperzia selago & $29^{2}$ & 24.4 & $7^{2}$ & - & $3^{2}$ & - \\
\hline lv & Vaccinium gaultherioides & $18^{7}$ & 40.8 & - & - & - & - \\
\hline $\mathrm{CC}$ & Avenula versicolor & $18^{2}$ & 40.8 & - & - & - & - \\
\hline Ns & Avenella flexuosa & $82^{3}$ & 3.2 & $96^{4}$ & 52.8 & $43^{3}$ & - \\
\hline & Sphagnum sp. div. $\left(\mathrm{E}_{0}\right)$ & $18^{2}$ & - & $35^{5}$ & 28.1 & $7^{2}$ & - \\
\hline & Dryopteris dilatata s. 1. & $7^{2}$ & - & $66^{3}$ & 18.0 & $58^{3}$ & - \\
\hline Aa, pe & Athyrium distentifolium & • & - & $43^{5}$ & 9.9 & $42^{5}$ & 3.7 \\
\hline ai & Oxalis acetosella & - & - & $33^{3}$ & - & $58^{4}$ & 27.9 \\
\hline Ns, MU & Gentiana asclepiadea & • & - & $26^{2}$ & - & $38^{2}$ & 15.3 \\
\hline ac, MU & Geranium sylvaticum & • & - & $4^{2}$ & - & $74^{3}$ & 75.1 \\
\hline ac, VP & Luzula sylvatica & - & - & $17^{2}$ & - & $67^{3}$ & 51.8 \\
\hline EA, (ai) & Rubus idaeus & • & - & $31^{3}$ & - & $66^{3}$ & 36.4 \\
\hline ss & Salix silesiaca & $\bullet$ & - & $17^{2}$ & - & $56^{3}$ & 43.5 \\
\hline & Hypericum maculatum & • & - & $8^{2}$ & - & $56^{2}$ & 54.4 \\
\hline ac, MU & Veratrum *lobelianum & $18^{1}$ & - & $26^{2}$ & - & $52^{2}$ & 26.1 \\
\hline ac, cy & Valeriana tripteris & $\bullet$ & - & $4^{2}$ & - & $50^{3}$ & 55.3 \\
\hline $\mathrm{Ns}, \mathrm{NC}$ & Soldanella carpatica & $18^{1}$ & - & $14^{2}$ & - & $47^{2}$ & 35.1 \\
\hline Fs & Polygonatum verticillatum & - & - & $5^{2}$ & - & $46^{2}$ & 50.5 \\
\hline MU & Aconitum firmum & • & - & $2^{2}$ & - & $44^{2}$ & 54.5 \\
\hline EA, fs & Senecio nemorensis agg. & - & - & $7^{2}$ & - & $42^{3}$ & 43.9 \\
\hline $\mathrm{pm}$ & Ribes petraeum & - & - & $5^{3}$ & - & $41^{5}$ & 46.4 \\
\hline $\mathrm{MC}$ & Viola biflora & - & - & $2^{2}$ & - & $41^{3}$ & 52.2 \\
\hline Aa, ac & Cicerbita alpina & - & - & $4^{2}$ & - & $38^{2}$ & 45.8 \\
\hline ai, MU & Primula elatior & - & - & $\bullet$ & - & $37^{2}$ & 53.5 \\
\hline & Alchemilla sp. div. & - & - & +1 & - & $36^{2}$ & 51.6 \\
\hline Aa & Adenostyles alliariae & - & - & $4^{2}$ & - & $35^{3}$ & 42.3 \\
\hline Fs & Paris quadrifolia & - & - & $1^{2}$ & - & $32^{2}$ & 47.2 \\
\hline ai, ac, po & Chaerophyllum hirsutum & - & - & $t^{2}$ & - & $31^{2}$ & 46.8 \\
\hline Fs & Daphne mezereum & - & - & - & - & $31^{2}$ & 48.9 \\
\hline Fs & Myosotis sylvatica & • & - & $t^{2}$ & - & $31^{2}$ & 46.8 \\
\hline $\mathrm{Aa}, \mathrm{ac}$ & Doronicum austriacum & - & - & $1^{2}$ & - & $30^{3}$ & 44.7 \\
\hline Ap & Leucanthemum rotundifolium & - & - & - & - & $29^{5}$ & 46.4 \\
\hline & Rosa pendulina & • & - & $2^{3}$ & - & $29^{3}$ & 41.4 \\
\hline $\mathrm{Aa}$ & Ranunculus platanifolius & - & - & $2^{2}$ & - & $26^{2}$ & 38.6 \\
\hline $\mathrm{cl}$, ai & Geum rivale & - & - & - & - & $26^{2}$ & 43.9 \\
\hline & Heracleum sphondylium & • & - & - & - & $24^{2}$ & 42.2 \\
\hline cy, ac & Cortusa matthioli & - & - & - & - & $24^{3}$ & 42.2 \\
\hline $\mathrm{Aa}, \mathrm{Fs}$ & Milium effusum & • & - & +1 & - & $24^{2}$ & 40.7 \\
\hline ai, po, $\mathrm{MC}$ & Chrysosplenium alternifolium & • & - & $1^{2}$ & - & $23^{3}$ & 38.4 \\
\hline & Lonicera nigra & • & - & $t^{2}$ & - & $23^{2}$ & 39.8 \\
\hline Fs, EA & Epilobium montanum & - & - & $1^{1}$ & - & $21^{2}$ & 36.5 \\
\hline Fs & Galeobdolon luteum s. 1. & $\bullet$ & - & $\bullet$ & - & $21^{2}$ & 39.5 \\
\hline Pinion mu & & & & & & & \\
\hline & Pinus mugo & $100^{8}$ & - & $100^{9}$ & - & $100^{8}$ & - \\
\hline LV & Vaccinium vitis-idaea & $79^{3}$ & 16.5 & $52^{3}$ & 1.7 & $43^{3}$ & - \\
\hline & Pleurozium schreberi $\left(\mathrm{E}_{0}\right)$ & $75^{5}$ & 20.2 & $45^{3}$ & 9.3 & $26^{3}$ & - \\
\hline $\mathrm{LV}$, pe & Vaccinium myrtillus & $68^{5}$ & - & $98^{6}$ & 30.9 & $81^{5}$ & - \\
\hline & Dicranum scoparium $\left(\mathrm{E}_{0}\right)$ & $57^{3}$ & - & $79^{5}$ & 16.6 & $66^{3}$ & - \\
\hline
\end{tabular}


TABLE 2. cont

\begin{tabular}{|c|c|c|c|c|c|c|c|}
\hline & & $\begin{array}{c}\text { Freq } \\
(\%)\end{array}$ & $\begin{array}{l}\text { Fide } \\
\text { (phi) }\end{array}$ & $\begin{array}{c}\text { Freq } \\
(\%)\end{array}$ & $\begin{array}{l}\text { Fide } \\
\text { (phi) }\end{array}$ & $\begin{array}{c}\text { Freq } \\
(\%)\end{array}$ & $\begin{array}{l}\text { Fide } \\
\text { (phi) }\end{array}$ \\
\hline & Hylocomium splendens $\left(\mathrm{E}_{0}\right)$ & $57^{5}$ & 6.8 & $49^{3}$ & 8.7 & $36^{3}$ & - \\
\hline NS, pe & Homogyne alpina & $32^{2}$ & - & $80^{3}$ & 20.7 & $69^{3}$ & - \\
\hline \multirow[t]{2}{*}{$\mathrm{Cv}$} & Calamagrostis villosa & $32^{2}$ & - & $79^{5}$ & 26.1 & $60^{5}$ & - \\
\hline & Juniperus sibirica & $29^{2}$ & 19.1 & $9^{2}$ & - & $6^{2}$ & - \\
\hline pe & Sorbus *glabrata & $4^{2}$ & - & $47^{3}$ & - & $77^{3}$ & 32.8 \\
\hline \multicolumn{8}{|c|}{ Piceion excelsae, Piceetalia excelsae, Vaccinio-Piceetea } \\
\hline & Picea abies & $18^{1}$ & - & $45^{2}$ & - & $56^{2}$ & 13.4 \\
\hline & Pinus cembra & $7^{2}$ & 2.0 & $5^{2}$ & - & $6^{2}$ & 2.7 \\
\hline & Barbilophozia lycopodioides $\left(\mathrm{E}_{0}\right)$ & $7^{2}$ & - & $15^{3}$ & 16.9 & $4^{3}$ & - \\
\hline
\end{tabular}

Fagetalia, Querco-Fagetea

fs Dryopteris filix-mas

fs, pt Phyteuma spicatum

fs, po Stellaria nemorum

fs $\quad$ Prenanthes purpurea

fs $\quad$ Gymnocarpium dryopteris

ai, MC Crepis paludosa

fs, (ai) Athyrium filix-femina

fs, VP Hieracium murorum

$\mathrm{cb} \quad$ Galium schultesii

ce, (ca) Rubus saxatilis

ac Polystichum lonchitis

ai, ac, de Thalictrum aquilegiifolium

Fs Mercurialis perennis

Caricetea curvulae, Loiseleurio-Vaccinietea

CC Polytrichum sp. div.

vm Polytrichum strictum $\left(\mathrm{E}_{0}\right)$

lv Calluna vulgaris

CC Agrostis rupestris

LV Empetrum nigrum s. 1.

$\mathrm{CC} \quad$ Cetraria nivalis

CC Cetraria cucullata

$\mathrm{fv}, \mathrm{SH} \quad$ Doronicum stiriacum

Adenostyletalia, Calamagrostiletalia villosae, Mulgedio-Aconitetea

$\begin{array}{ll}\mathrm{Cv} & \text { Luzula luzuloides } \\ \mathrm{Cv} & \text { Solidago *minuta } \\ \mathrm{Cv} & \text { Gentiana punctata } \\ \mathrm{aa} & \text { Oreogeum montanum } \\ \mathrm{pt}, \mathrm{Mo}, \mathrm{MU} & \text { Bistorta major } \\ \mathrm{Cv} & \text { Campanula tatrae } \\ \mathrm{Cv}, \mathrm{ac} & \text { Acetosa arifolia } \\ \mathrm{Cv}, \mathrm{ce} & \text { Cirsium erisithales } \\ \mathrm{MU}, \text { ac } & \text { Senecio subalpinus } \\ \mathrm{ca} & \text { Calamagrostis arundinacea } \\ \mathrm{cr} & \text { Calamagrostis varia } \\ \mathrm{fc} & \text { Festuca carpatica } \\ \mathrm{Cv} & \text { Cyanus mollis } \\ \mathrm{Cv} & \text { Pimpinella major } \\ \mathrm{MU}, \text { na } & \text { Poa chaixii } \\ \mathrm{po} & \text { Valeriana } * \text { sambucifolia } \\ \mathrm{de}, \mathrm{cu} & \text { Epilobium alpestre }\end{array}$

$11^{2}$

$32^{1}$

$25^{2}$

$11^{1}$

$14^{2}$

$7^{3}$

•

\section{Other taxa}

$\begin{array}{ll}\text { Ns } & \text { Ligusticum mutellina } \\ \text { EA } & \text { Chamerion angustifolium } \\ & \text { Hylotelephium argutum } \\ & \text { Saxifraga rotundifolia } \\ \mathrm{Ns}, \mathrm{Cv} & \begin{array}{l}\text { Potentilla aurea } \\ \text { Digitalis } \text { grandiflora }\end{array} \\ \mathrm{EA} & \text { Fragaria vesca } \\ \text { ar, (de) } & \text { Arabis alpina } \\ \mathrm{GU} & \text { Urtica dioica } \\ & \text { Senecio } * \text { carniolicus }\end{array}$

\begin{tabular}{|c|c|}
\hline • & - \\
\hline & - \\
\hline & - \\
\hline & - \\
\hline & - \\
\hline & - \\
\hline & - \\
\hline & - \\
\hline & - \\
\hline & - \\
\hline & - \\
\hline & - \\
\hline & - \\
\hline
\end{tabular}

30.2

35.0

29.8

16.9

23.2

28.4

19.2

22.3

25.9

25.9

31.5

29.2

26.8

$\begin{array}{lccccc}25^{3} & 2.4 & 32^{3} & 22.5 & 11^{3} & - \\ 21^{2} & 18.2 & 7^{3} & 4.3 & 1^{2} & - \\ 11^{2} & 15.1 & 2^{2} & - & 2^{2} & - \\ 18^{2} & 36.6 & +^{2} & - & \cdot & - \\ 14^{4} & 20.1 & 3^{3} & - & \cdot & - \\ 11^{2} & 26.5 & +^{1} & - & \cdot & - \\ 11^{3} & 31.5 & - & - & \text {. } \\ 11^{2} & 31.5 & - & - & \text {. }\end{array}$




\begin{tabular}{|c|c|c|c|c|c|c|c|}
\hline & & $\begin{array}{c}\text { Freq } \\
(\%)\end{array}$ & $\begin{array}{l}\text { Fide } \\
\text { (phi) }\end{array}$ & $\begin{array}{c}\text { Freq } \\
(\%)\end{array}$ & $\begin{array}{l}\text { Fide } \\
\text { (phi) }\end{array}$ & $\begin{array}{c}\text { Freq } \\
(\%)\end{array}$ & $\begin{array}{l}\text { Fide } \\
\text { (phi) }\end{array}$ \\
\hline SH & Luzula alpinopilosa & $11^{2}$ & 16.5 & $2^{3}$ & - & $\bullet$ & - \\
\hline \multicolumn{8}{|c|}{ Bryophytes and Lichens $\left(\mathbf{E}_{0}\right)$} \\
\hline & Plagiothecium curvifolium & $14^{2}$ & - & $11^{2}$ & 19.8 & $4^{2}$ & - \\
\hline & Ptilidium ciliare & $14^{2}$ & 8.5 & $6^{2}$ & - & $6^{2}$ & - \\
\hline & Rhytidiadelphus triquetrus & $7^{2}$ & - & $8^{3}$ & - & $25^{3}$ & 22.9 \\
\hline & Drepanocladus uncinatus & $7^{1}$ & 2.0 & $1^{2}$ & - & $14^{3}$ & 24.7 \\
\hline & Racomitrium lanuginosum & $18^{1}$ & 36.6 & $t^{3}$ & - & • & - \\
\hline & Cladonia stellaris & $14^{3}$ & 31.9 & $t^{2}$ & - & $\bullet$ & - \\
\hline & Cladonia bellidiflora & $11^{2}$ & 15.1 & $3^{2}$ & - & • & - \\
\hline & Anastrepta orcadensis & $11^{1}$ & 23.0 & $1^{3}$ & - & • & - \\
\hline & Polytrichum piliferum & $11^{2}$ & 26.5 & $t^{3}$ & - & $\bullet$ & - \\
\hline & Plagiothecium denticulatum & • & - & $6^{2}$ & - & $19^{2}$ & 22.5 \\
\hline & Rhytidiadelphus squarrosus & • & - & $6^{3}$ & - & $10^{3}$ & 9.3 \\
\hline & Brachythecium reflexum & • & - & $4^{2}$ & - & $10^{3}$ & 13.7 \\
\hline & Cladonia uncialis & $11^{2}$ & 31.5 & - & - & $\bullet$ & - \\
\hline & Pseudevernia furfuracea & $11^{2}$ & 31.5 & • & - & • & - \\
\hline & Thamnolia vermicularis & $11^{2}$ & 31.5 & • & - & $\bullet$ & - \\
\hline
\end{tabular}

Explanations:

CC - Caricetea curvulae; EA - Epilobietea angustifolii; GU - Galio-Urticetea; LV - Loiseleurio-Vaccinietea; MC - Montio-Cardaminetea; MU Mulgedio-Aconitetea; SH - Salicetea herbace; VP - Vaccinio-Piceetea; Aa - Adenostyletalia; Ap - Athyrio-Piceetalia; Cv - Calamagrostietalia villosae; Fs - Fagetalia sylvaticae; Mo - Molinietalia; Ns - Nardetalia strictae; aa - Adenostylion; ac - Acerenion pseudoplatani; ai - Alnion incanae; ar - Arabidion alpinae; ca - Calamagrostion arundinaceae; $\mathbf{c b}$ - Carpinion betuli; ce - Cephalanthero-Fagenion; cl - Calthion; cr - Calamagrostion variae; cu - Carduo-Urticion dioicae; cy - Cystopteridion; de - Delphinenion elati; fc - Festucion carpaticae; fs - Fagion sylvaticae; fv - Festucion versicoloris; lv Loiseleurio-Vaccinion; na - Nardo-Agrostion tenuis; pe - Piceion excelsae; po - Petasition; pt - Polygono-Trisetion; ss - Salicion silesiacae; vm - Vaccinion myrtilli; (ai) - taxon with lower relation to the relevant syntaxon and with coenological optimum also in other syntaxon.

\section{Ordination}

The detrended correspondence analysis (DCA) ordination diagram of 341 relevés shows the major differences in the direction of the first axis (length of gradients: 4.059; eigenvalue: 0.444 , see also Table 3 ) owing to the different content of available nutrients in the soil, correlated with soil-base status and temperature. In the direction of the second axis (length of gradients: 2.655; eigenvalue: 0.220) the major gradient in species composition was associated with soil moisture (Fig. 2). Light availability is negatively correlated with moisture, possibly due to the development of higher denser stands of broad-leaved herbs in moist habitats, which decrease light availability near the soil surface. Individual clusters differ markedly in their affinity to major ecological factors. On the left side of the graph (Fig. $5)$, there are relevés from the extremely rough sites, in the transition from the subalpine belt to the alpine. The occurrence of species like Empetrum nigrum s. 1., Juncus trifidus, Vaccinium gaultherioides etc., positively correlated with light available and negatively with moisture (Fig. 2), is connected above all with the extreme site conditions. An important factor is also the small hight of dwarf pine. The branches of Pinus mugo are spread near the ground and they do not create a great light barier. Therefore, the light can penetrate into the herb layer. The species of acid-oligotrophic character from the middle part of Fig. 2 indicate biotopes with thicker layers of undecomposed humus, where acidophilous and sciophilous species predominated. On the right side of Fig. 2, we can see the species that occur on nutrient-rich soils (primarily, with high contents of nitrogen), showing close syngenetic relation with plant communities of the class Mulgedio-Aconitetea. This fact points the sites with favourable edaphic properties and good de- composition of humus. Non-calcareous substrata, e.g. in protected areas, are occupied by predominantly wet (periodically flooded) biotopes, where the increased moisture, provided by the oxygenated streaming water, supports the nitrification and humification processes.

Changeovers between the groups of relevés (Fig. 5) are smooth, because a decisive factor - available nutrients and moisture related with the thickness of the layer of decomposed humus - increasing in specific conditions, decreases mutual differences between individual communities.

The group of relevés from the Fig. 5, marked by diamonds, represents the subassociation Adenostylo alliariaePinetum mugo seslerietosum calcariae, described by Šoltésová (1974) from the stands on dry parts of calcareous rocks and screes as "considerably different ecological-floristic conditions". These are negatively correlated with moisture and positively with continentality. It would be appropriate to classify this type of dwarf pine vegetation at the level of separate association (Bernátová and Uhlířová, in prep.).

\section{Syntaxonomy and nomenclature}

Roso pendulinae-Pinetea mugo Theurillat in Theurillat et al. 1995

Prostrate (subalpine shrubs) or erected (woodland-like, $<8$ m) communities dominated by Pinus mugo s. 1. both on basic and acid substrata.

\section{Junipero-Pinetalia mugo Boşcaiu 1971}

Pinion mugo Pawłowski in Pawłowski et al. 1928

Nomenclatural type: Pinetum mughi carpaticum Pawłowski in Pawłowski et al. 1928 (holotypus).

Inclusive: Pinion mughi (Pawłowski et al. 1928; Klika 1947) Šoltésová 1974 (art. 31). 


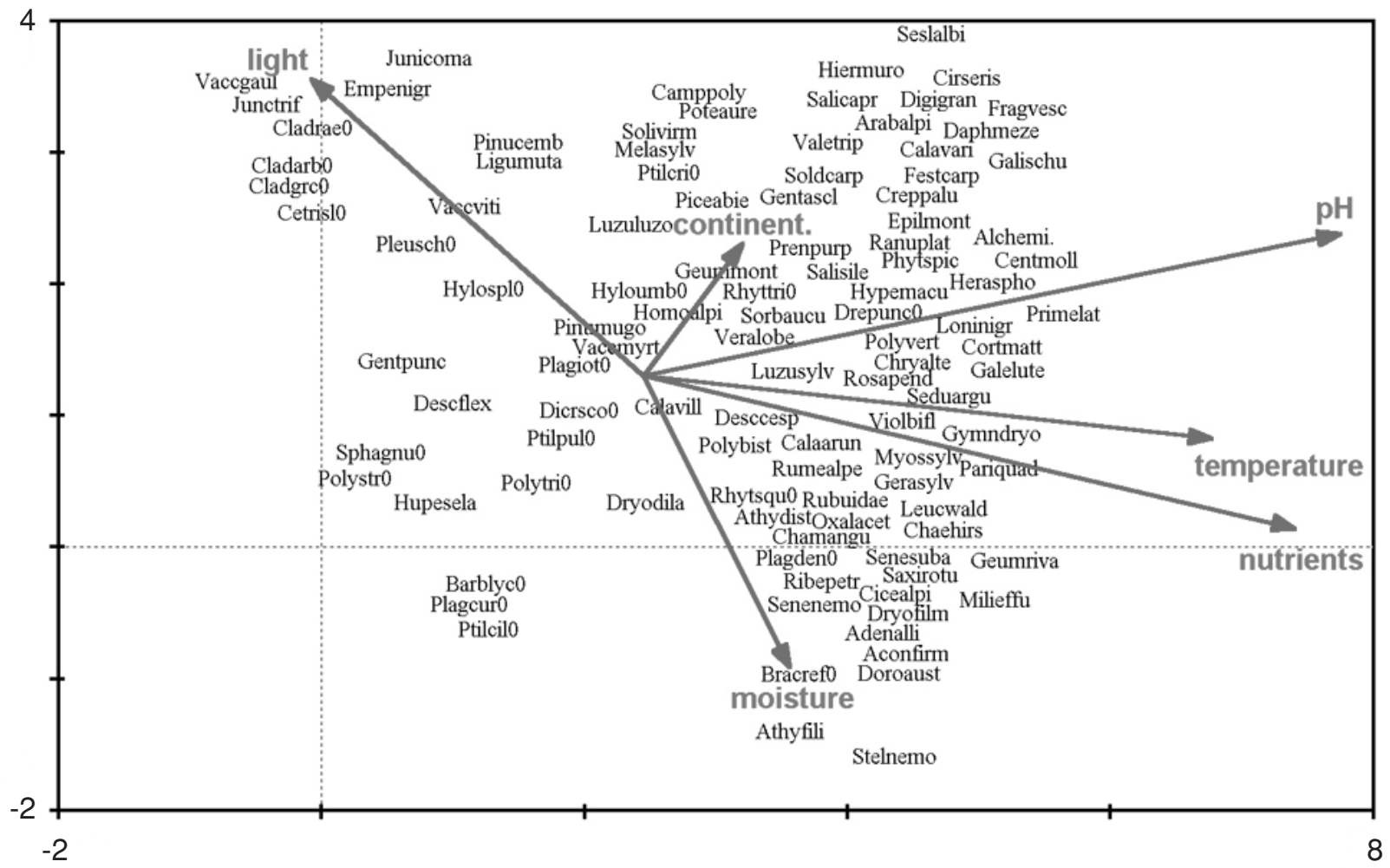

Fig. 2. Detrended correspondence analysis (DCA) ordination diagram of Pinion mugo data set. Ordination scores of the most important species (weight $>1 \%$ ). Species list: Aconfirm - Aconitum firmum, Adenalli - Adenostyles alliariae, Alchemi. - Alchemilla sp. div., Arabalpi - Arabis alpina, Athyfili - Athyrium filix-femina, Athydist - Athyrium distentifolium, Barblyc0 - Barbilophozia lycopodioides $\left(\mathrm{E}_{0}\right)$, Bracref0 - Brachythecium reflexum ( $\left.\mathrm{E}_{0}\right)$, Calaarun - Calamagrostis arundinacea, Calavari - Calamagrostis varia, Calavill - Calamagrostis villosa, Campoly - Campanula tatrae, Centmoll - Cyanus mollis, Cetris10 - Cetraria islandica $\left(\mathrm{E}_{0}\right)$, Chaehirs - Chaerophyllum hirsutum, Chamangu - Chamerion angustifolium, Chryalte - Chrysosplenium alternifolium, Cicealpi - Cicerbita alpina, Cirseris - Cirsium erisithales, Cladarb0 - Cladonia arbuscula $\left(\mathrm{E}_{0}\right)$, Cladgrc0 - Cladonia gracilis ( $\left.\mathrm{E}_{0}\right)$, Cladrae0 - Cladonia rangiferina $\left(\mathrm{E}_{0}\right)$, Cortmatt - Cortusa matthioli, Creppalu - Crepis paludosa, Daphmeze - Daphne mezereum, Desccesp - Deschampsia cespitosa, Descflex - Avenella flexuosa, Dicrsco0 - Dicranum scoparium $\left(\mathrm{E}_{0}\right)$, Digigran - Digitalis grandiflora, Doroaust - Doronicum austriacum, Drepunc0 - Sanionia uncinata $\left(\mathrm{E}_{0}\right)$, Dryodila - Dryopteris dilatata s. 1., Dryofilm - Dryopteris filix-mas, Empenigr - Empetrum nigrum s. 1., Epilmont - Epilobium montanum, Festcarp - Festuca carpatica, Fragvesc - Fragaria vesca, Galelute - Galeobdolon luteum s. 1., Galischu - Galium schultesii, Gentascl - Gentiana asclepiadea, Gentpunc - Gentiana punctata, Gerasylv - Geranium sylvaticum, Geummont - Oreogeum montanum, Geumriva - Geum rivale, Gymndryo - Gymnocarpium dryopteris, Heraspho - Heracleum sphondylium, Hiermuro - Hieracium murorum, Homoalpi - Homogyne alpina, Hylospl0 - Hylocomium splendens ( $\mathrm{E}_{0}$ ), Hyloumb0 - Hylocomium umbratum $\left(\mathrm{E}_{0}\right)$, Hypemacu - Hypericum maculatum, Hupesela - Huperzia selago, Junctrif - Juncus trifidus, Junicoma - Juniperus sibirica, Leucwald - Leucanthemum rotundifolium, Ligumuta - Ligusticum mutellina, Loninigr - Lonicera nigra, Luzuluzo - Luzula luzuloides, Luzusylv - Luzula sylvatica, Mela sylv - Melampyrum sylvaticum, Milieffu - Milium effusum, Myossylv - Myosotis sylvatica, Oxalacet - Oxalis acetosella, Pariquad - Paris quadrifolia, Phytspic - Phyteuma spicatum, Piceabie - Picea abies, Pinucemb - Pinus cembra, Pinumugo - Pinus mugo, Plagcur0 - Plagiothecium curvifolium $\left(\mathrm{E}_{0}\right)$, Plagden0 - Plagiothecium denticulatum $\left(\mathrm{E}_{0}\right)$, Plagiot0 - Plagiothecium sp. $\left(\mathrm{E}_{0}\right)$, Pleusch0 - Pleurozium schreberi $\left(\mathrm{E}_{0}\right)$, Polybist - Bistorta major, Polystr0 - Polytrichum strictum $\left(\mathrm{E}_{0}\right)$, Polytri0 - Polytrichum sp. div. ( $\left.\mathrm{E}_{0}\right)$, Polyvert - Polygonatum verticillatum, Poteaure - Potentilla aurea, Prenpurp - Prenanthes purpurea, Primelat - Primula elatior, Ptilcilo - Ptilidium ciliare $\left(\mathrm{E}_{0}\right)$, Ptilpul0 - Ptilidium pulcherrimum, Ptilcri0 - Ptilium crista-castrensis $\left(\mathrm{E}_{0}\right)$, Ranuplat - Ranunculus platanifolius, Rhyttri0 - Rhytidiadelphus squarrosus $\left(\mathrm{E}_{0}\right)$, Rhyttri0 - Rhytidiadelphus triquetrus $\left(\mathrm{E}_{0}\right)$, Ribepetr - Ribes petraeum, Rosapend - Rosa pendulina, Rubuidae - Rubus idaeus, Rumealpe - Acetosa arifolia, Salicapr - Salix caprea, Salisile - Salix silesiaca, Saxirotu - Saxifraga rotundifolia, Seduargu - Hylotelephium argutum, Senenemo - Senecio nemorensis agg., Senesuba - Senecio subalpinus, Seslalbi - Sesleria albicans, Stelnemo - Stellaria nemorum, Soldcarp - Soldanella carpatica, Solivirm - Solidago * minuta, Sorbaucu - Sorbus aucuparia, Sphagnu0 - Sphagnum sp. div. ( $\left.\mathrm{E}_{0}\right)$, Vaccgaul - Vaccinium gaultherioides, Vaccmyrt - Vaccinium myrtillus, Vaccviti - Vaccinium vitis-idaea, Valetrip Valeriana tripteris, Veralobe - Veratrum * lobelianum, Violbifl - Viola biflora.

Synonyms: Pinion mughi Pawłowski 1928 (art. 2b, 8), Vaccinion myrtilli Krajina 1933 p.p. (art. 29b), MyrtilloPiceion excelsae Březina et Hadač in Hadač 1962 p.p. (art. 31), Mughion Domin 1923 (nom. nud.).

Syntaxonomical synonym: Athyrio alpestris-Pinion mughi Jirásek 1996.

Dwarf pine stands in the supramontanous and subalpine belts on siliceous and calcareous bedrocks in the Carpathians.

\section{Cetrario islandicae-Pinetum mugo Hadač 1956}

Table 2, column A, Fig. 6

Nomenclatural type: Hadač 1956: 23, Table 4, rel. 17, lectotypus hoc loco.
Original form of the name: Cetrario-pinetum mughi Hadač 1956.

Inclusive: Vaccinio myrtilli-Pinetum mughi cladonietosum rangiferino-gracilis Šoltésová 1974 p.p. (art. 13)*.

Synonyms: Pinetum mughi tatrense lichenoso-muscosum Krajina 1933 (art. 34a), Mughetum tatrense lichenoso-muscosum Dostál 1935 (art. 2b, 31).

Non: Mughetum myrtilletoso-sphagnosum Sillinger 1933 , Sphagno-Mughetum Mráz 1956.

* Original form of the name, mentioned by author in her work is Vaccinio myrtilli-Pinetum mughi cladonietosum rangiferino-gracilis (Krajina 1934) Šoltésová 1974. 


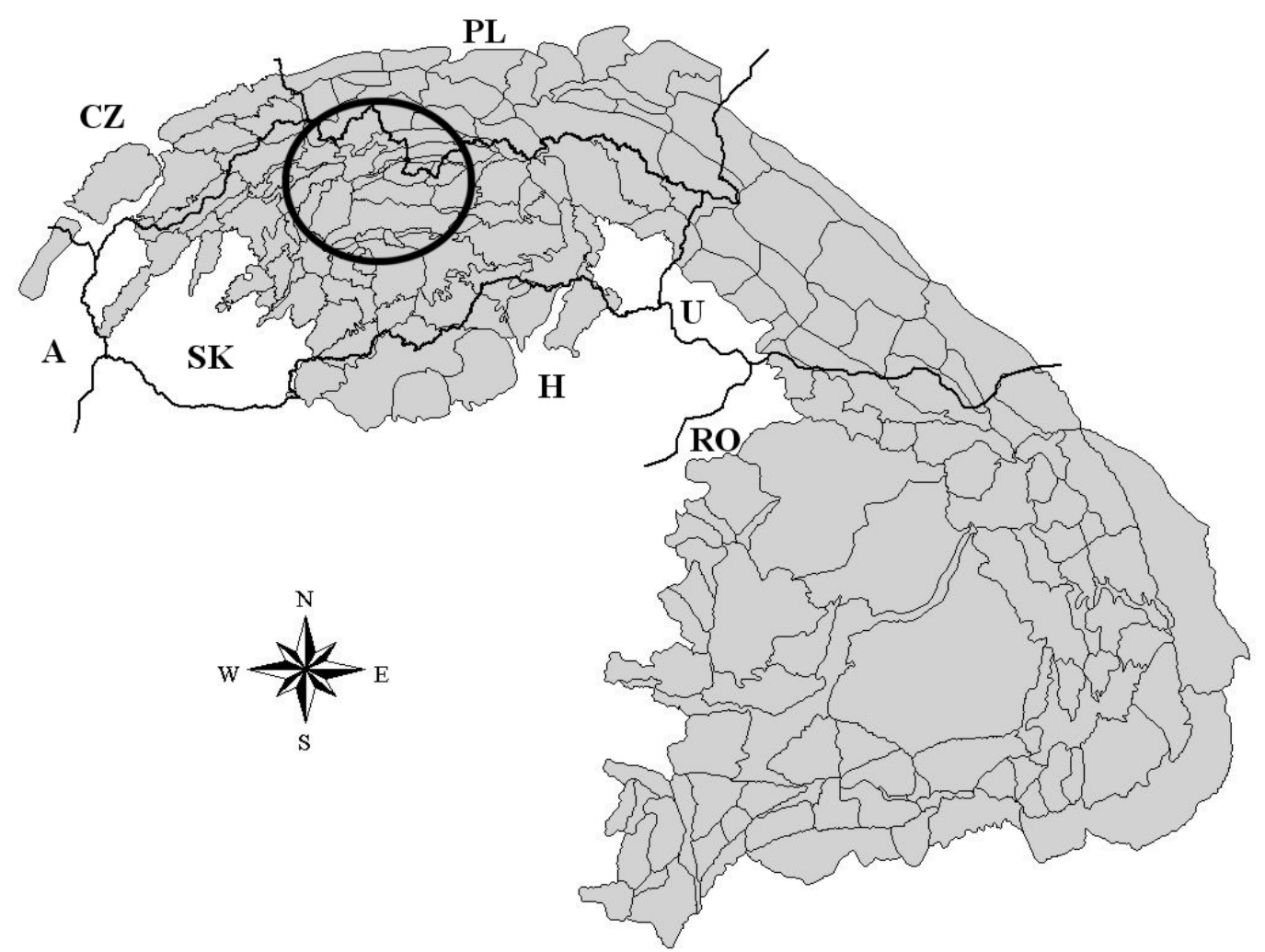

Fig. 3. Area of occurrence of dwarf pine stands (marked by ellipse) in the Western Carpathians. Grey area stands for orographical units in the Carpathians.

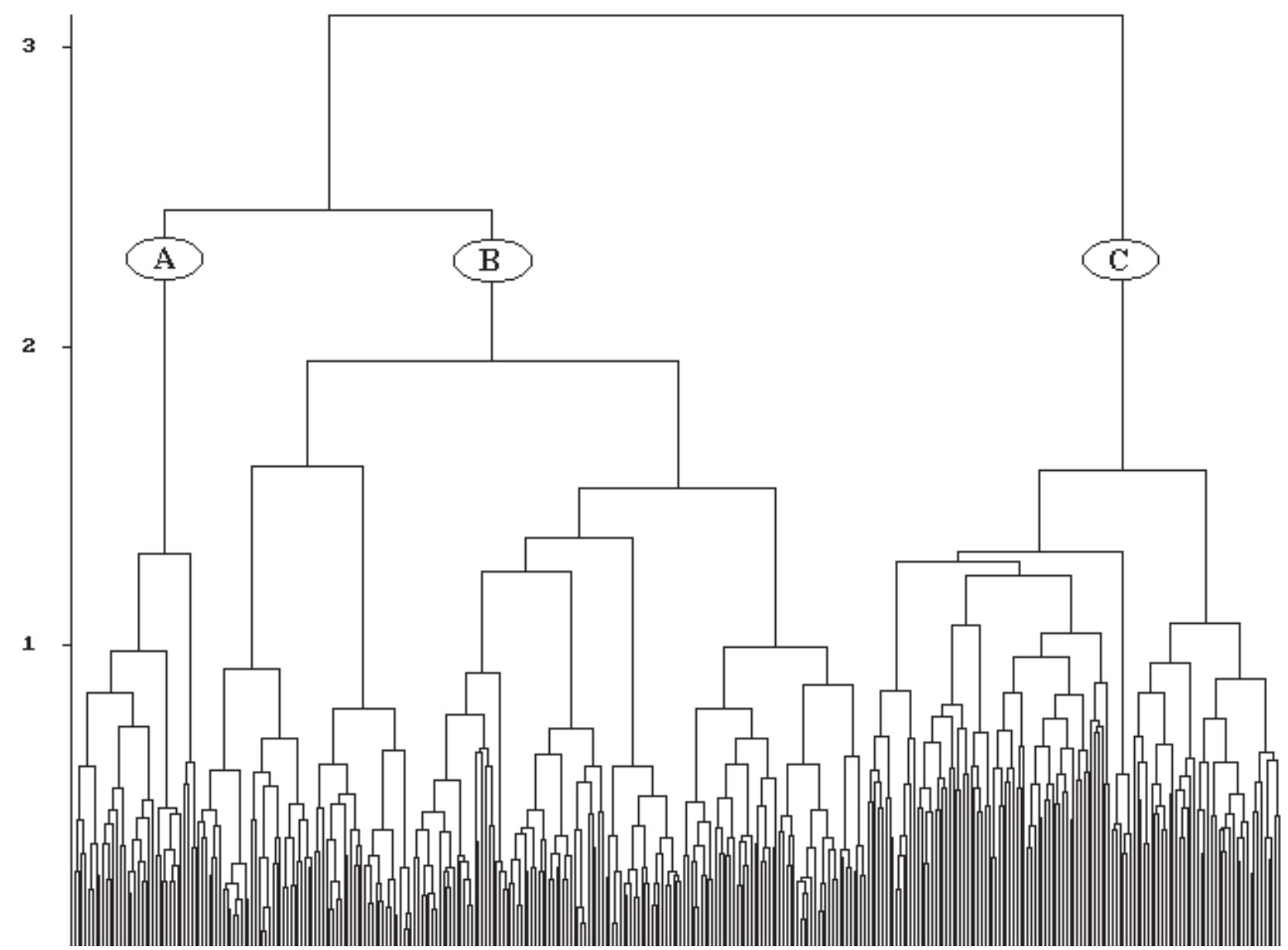

Fig. 4. Dendrogram of numerical classification of plant communities of the alliance Pinion mugo in the Western Carpathians. On its base the synoptic table was formed, however, the problematic relevés were re-shuffled.

A - Cetrario islandicae-Pinetum mugo, B - Homogyno alpinae-Pinetum mugo, C - Adenostylo alliariae-Pinetum mugo (used parameters: $\beta$-flexible method with Wishart's similarity coefficient). 
TABLE 3. Weighted correlation matrix for the environmental factors from the DCA ordination.

\begin{tabular}{|c|c|c|c|c|c|c|c|c|}
\hline \multicolumn{9}{|c|}{ Weighted correlation matrix $($ weight $=$ sample total $)$} \\
\hline SPEC AX1 & 1.0000 & & & & & & & \\
\hline SPEC AX2 & -0.2682 & 1.0000 & & & & & & \\
\hline SPEC AX3 & -0.0557 & 0.0626 & 1.0000 & & & & & \\
\hline SPEC AX4 & 0.0205 & 0.0324 & -0.0628 & 1.0000 & & & & \\
\hline ENVI AX1 & 0.9628 & -0.2622 & -0.0568 & 0.0394 & 1.0000 & & & \\
\hline ENVI AX2 & -0.2836 & 0.8902 & 0.0393 & 0.0050 & -0.2945 & 1.0000 & & \\
\hline ENVI AX3 & -0.0671 & 0.0429 & 0.8159 & -0.1074 & -0.0697 & 0.0481 & 1.0000 & \\
\hline ENVI AX4 & 0.0495 & 0.0058 & -0.1143 & 0.7671 & 0.0514 & 0.0066 & -0.1400 & 1.0000 \\
\hline Light & -0.4924 & 0.5953 & -0.3863 & -0.1302 & -0.5115 & 0.6688 & -0.4735 & -0.1698 \\
\hline Tempera & 0.7611 & -0.2949 & 0.3592 & -0.0923 & 0.7906 & -0.3313 & 0.4403 & -0.1204 \\
\hline Contine & 0.1064 & 0.1814 & -0.1498 & 0.6095 & 0.1105 & 0.2037 & -0.1836 & 0.7945 \\
\hline Moistur & 0.2455 & -0.5237 & -0.1244 & -0.2930 & 0.2550 & -0.5883 & -0.1525 & -0.3820 \\
\hline $\mathrm{pH}$ & 0.8942 & -0.0057 & -0.2269 & 0.0298 & 0.9288 & -0.0064 & -0.2781 & 0.0389 \\
\hline Nutrien & 0.8872 & -0.4686 & -0.2473 & 0.0972 & 0.9215 & -0.5264 & -0.3031 & 0.1268 \\
\hline Light & 1.0000 & & & & & & & \\
\hline Tempera & -0.6142 & 1.0000 & & & & & & \\
\hline Contine & 0.0504 & 0.0453 & 1.0000 & & & & & \\
\hline Moistur & -0.3445 & 0.2993 & -0.0877 & 1.0000 & & & & \\
\hline $\mathrm{pH}$ & -0.2162 & 0.5705 & 0.1339 & 0.1049 & 1.0000 & & & \\
\hline \multirow[t]{2}{*}{ Nutrien } & -0.4929 & 0.6171 & 0.0490 & 0.2808 & 0.8372 & 1.0000 & & \\
\hline & Light & Tempera & Contine & Moistur & $\mathrm{pH}$ & Nutrien & & \\
\hline $\mathrm{N}$ & name & (weighted) mean & \multicolumn{2}{|c|}{ standard deviation } & \multicolumn{2}{|c|}{ inflation factor } & & \\
\hline 1 & SPEC AX1 & 2.4522 & \multicolumn{2}{|c|}{0.8637} & & & & \\
\hline 2 & SPEC AX2 & 1.3005 & \multicolumn{2}{|c|}{0.4603} & & & & \\
\hline 3 & SPEC AX3 & 0.9362 & \multicolumn{2}{|c|}{0.3963} & & & & \\
\hline 4 & SPEC AX4 & 1.0761 & \multicolumn{2}{|c|}{0.2804} & & & & \\
\hline 7 & ENVI AX3 & 0.9362 & \multicolumn{2}{|c|}{0.3234} & & & & \\
\hline 8 & ENVI AX4 & 1.0761 & \multicolumn{2}{|c|}{0.2151} & & & & \\
\hline 1 & Light & 5.7478 & \multicolumn{2}{|c|}{0.4732} & \multicolumn{2}{|c|}{2.3221} & & \\
\hline 2 & Tempera & 3.4099 & \multicolumn{2}{|c|}{0.3449} & \multicolumn{2}{|c|}{2.4324} & & \\
\hline 3 & Contine & 3.6725 & \multicolumn{2}{|c|}{0.2792} & \multicolumn{2}{|c|}{1.0372} & & \\
\hline 4 & Moistur & 5.6101 & \multicolumn{2}{|c|}{0.5177} & \multicolumn{2}{|c|}{1.2178} & & \\
\hline 5 & $\mathrm{pH}$ & 4.0544 & \multicolumn{2}{|c|}{1.1635} & \multicolumn{2}{|c|}{5.0078} & & \\
\hline 6 & Nutrien & 3.8229 & \multicolumn{2}{|c|}{1.0135} & \multicolumn{2}{|c|}{5.3888} & & \\
\hline
\end{tabular}

Diagnostic taxa: Pinus mugo (dom.), Avenula versicolor (dif.), Campanula alpina (dif.), Festuca supina (dif.), Hieracium alpinum agg. (dif.), Huperzia selago (dif.), Juncus trifidus (dif.), Oreochloa disticha (dif.), Pulsatilla scherfelii (dif.), Alectoria ochroleuca (dif.), Cetraria islandica (dif.), Cladonia arbuscula (dif.), C. gracilis (dif.), C. rangiferina (dif.), Vaccinium gaultherioides (dif.), Avenella flexuosa (const.), Vaccinium myrtillus (const.), V. vitis-idaea (const.), Pleurozium schreberi (const.).

\section{Homogyne alpinae-Pinetum mugo (Sillinger 1933) nom.} nov. hoc loco

Table 2, column B, Fig. 6

Nomenclatural type: Sillinger 1933: 123, rel. 1, lectotypus hoc loco.

Basionym: Mughetum myrtilletosum Sillinger 1933 (art. 34a).

Inclusive: Mughetum myrtilletosum Sillinger 1933, Mughetum myrtilletoso-calamagrostidetosum villosae Sillinger 1933 (art. 2b, 13), Mughetum myrtilletoso-sphagnosum Sillinger 1933 (art. 2b, 13), Vaccinietum myrtilli tatricum pinetosum mughi Krajina 1933 (art. 29b, 34a), Mughetum myrtilletosum Dostál 1935 (art. 2b, 31), Mughetum myrtilletosum Samek et al. 1957 (art. 31), Vaccinio myrtilli-Pinetum mughi cladonietosum rangiferino-gracilis Šoltésová 1974 p.p. (art. 13).

Synonyms: Pinetum montanae fruticosae Szafer et al., 1923 (art. 34a), Pinetum montanae Klika 1926 typ Vaccinietum (art. 3c, 31), Pinetum mughi (= Mughetum) carpaticum Pawłowski in Szafer et al. 1927 p.p. (art. 34a), Pinetum mughi carpaticum silicicolum Pawłowski in Szafer et al.1927 p.p. maj. (art. 34a), Pinetum mughi carpaticum Pawłowski in Pawłowski et al. 1928 p.p. (art. 34a), Pinetum mughi carpaticum silicicolum Pawłowski in Pawłowski et al. 1928 p.p. maj. (art. 31, 34a), Pinetum mughi carpaticum Walas 1933 p.p. (art. 31, 34a), Pinetum mughi carpaticum silicicolum Walas 1933 (art. 31, 34a), Pinetum mughi carpaticum calcicolum Walas 1933 p.p. min. (art. 34a), Myrtilleto-Pinetum mughi Hadač 1956 (art. 31), Sphagno-mughetum Mráz 1956 (art. 3f), Mughetum (=Pinetum mughi) carpaticum Pawłowski 1956 p.p. (art. 2b, 31, 34a), Myrtillo-Pinetum mughi Jeník 1961 (art. 31), Myrtillo-Pinetum mughi Šoltés 1969 (art. 1, 31), Vaccinio myrtilli-Pinetum mughi (Sillinger 1933) Šoltésová 1974 (art. 31), Vaccinio myrtilli- 


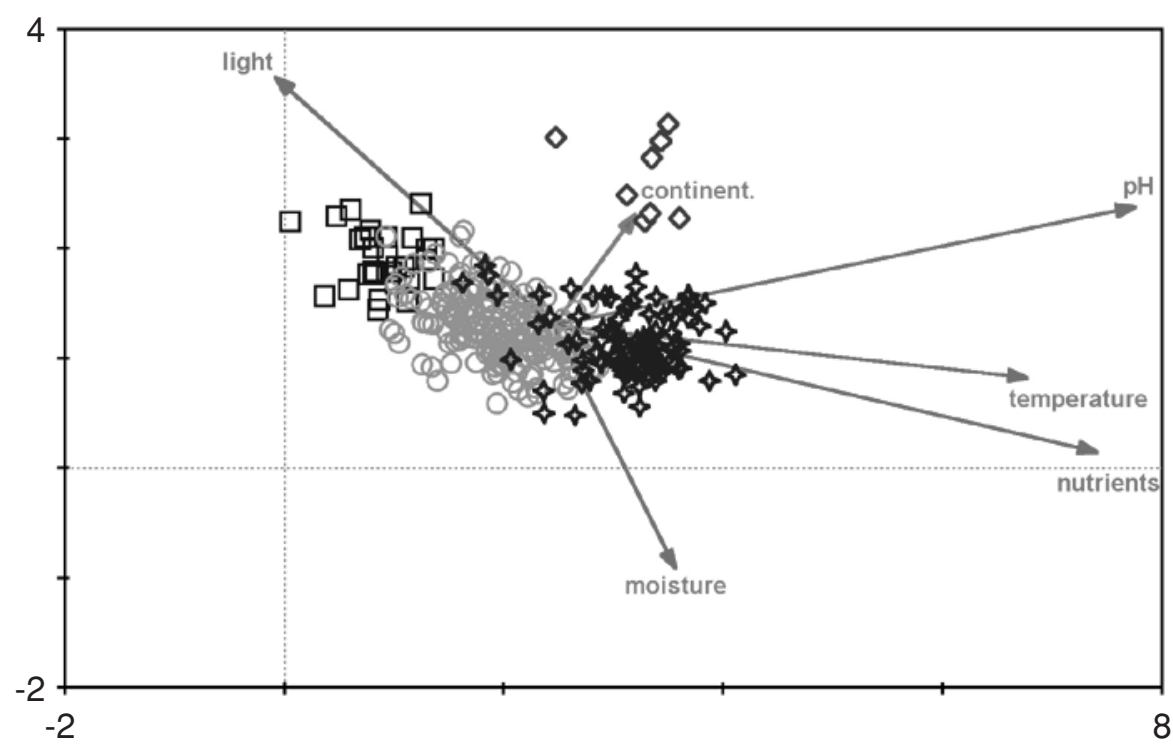

Fig. 5. Detrended correspondence analysis (DCA) ordination diagram of 341 relevés of the alliance Pinion mugo, based on average Ellenberg indicator values.

Squares $(\square)$ - Cetrario islandicae-Pinetum mugo, Circles $(\bigcirc)$ - Homogyno alpinaePinetum mugo, Stars (৬) - Adenostylo alliariae-Pinetum mugo (incl. Diamonds $(\diamond)$ - A.-P. seslerietosum calcariae).

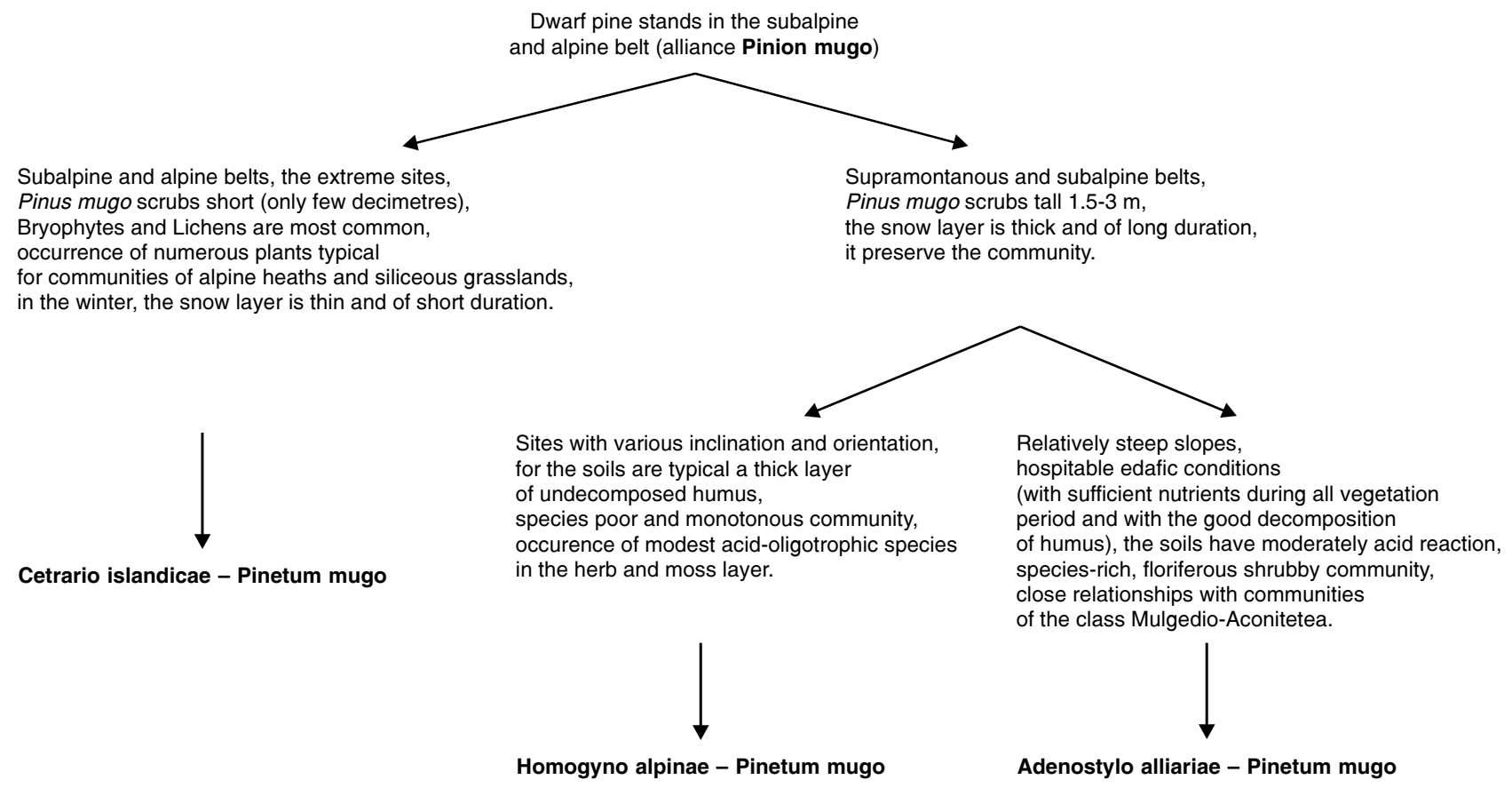

Fig. 6. Schematic division of associations of the alliance Pinion mugo.

Pinetum mugo Hadač 1956 (art. 31), Mughetum (silicicolum) myrtilloso-graminosum Domin 1923 (nom. nud.), Pinetum mughi carpaticum Pawłowski 1928 (nom. nud.).

Syntaxonomical synonym: Dryopterido-Pinetum mughi Unar in Unar et al. 1985.

Phantom name: Vaccinio myrtilli-Pinetum mughi sphagnetosum Sillinger 1933 (in Šomšák et al. 1981), Vaccinio myrtilli-Pinetum mughi Sillinger 1933 (in Unar et al. 1984, 1985), Sphagno nemorei-Pinetum mughi (Sillinger 1933) Mráz 1956 emend. Unar in Unar et al. 1984 (in Unar et al. 1984, 1985), Vaccinio myrtilli-Pinetum mugi (Pawłowski et al. 1928) Hadač 1956 (in Černušáková 1992).

Non: Vaccinio myrtilli-Pinetum montanae Morton 1927.

Diagnostic taxa: Pinus mugo (dom.), Athyrium distentifolium (dif. $\left.{ }^{1}\right)$, Dryopteris dilatata $\left(\operatorname{dif}^{1}\right)$, Gentiana asclepiadea (dif. ${ }^{1}$ ), Oxalis acetosella (dif. ${ }^{1}$ ), Avenella flexuosa (dif. $\left.{ }^{2}\right)$, Sphagnum sp. div. $\left(\right.$ dif $\left.^{2}\right)$, Calamagrostis villosa (const.), Homogyne alpina (const.), Vaccinium myrtillus (const.), Dicranum scoparium (const.).

${ }^{1}$ differential taxa against the association Cetrario islandicae-Pinetum mugo ${ }^{2}$ differential taxa against the association Adenostylo alliariae-Pinetum mugo

\section{Adenostylo alliariae-Pinetum mugo (Sillinger 1933)} Šoltésová 1974

Table 2, column C, Fig. 6

Nomenclatural type: Sillinger 1933: 120, rel. 2, lectotypus hoc loco.

Basionym: Mughetum altherbosum Sillinger 1933 (art. 34a).

Inclusive: Pinetum montanae Klika 1926 typ Calamagrostis villosa (art. 3c), Myrtilleto-Calamagrostidetum villosae carpaticum pinetosum mughi Krajina 1933 (art. 29b, 34a), 
Mughetum altherbosum calcicolum Sillinger 1933 (art. 34a), Mughetum altherbosum silicicolum Sillinger 1933 (art. 34a).

Synonyms: Pinetum montanae fruticosae Klika 1927 (art. 34a), Pinetum mughi (= Mughetum) carpaticum Pawłowski in Szafer et al. 1927 p.p. (art. 34a), Pinetum mughi carpaticum silicicolum Pawłowski in Szafer et al. 1927 p.p. min. (art. 34a), Pinetum mughi carpaticum calcicolum Pawłowski in Szafer et al. 1927 (art. 34a), Pinetum mughi carpaticum Pawłowski in Pawłowski et al. 1928 p.p. (art. 34a), Pinetum mughi carpaticum calcicolum Pawłowski in Pawłowski et al. 1928 (art. 31, 34a), Pinetum mughi carpaticum silicicolum Pawłowski in Pawłowski et al. 1928 p.p. min. (art. 31, 34a), Pinetum mughi carpaticum Walas 1933 p.p. (art. 31, 34a), Pinetum mughi carpaticum calcicolum Walas 1933 p.p. maj. (art. 31, 34a), Mughetum altherbosum calcicolum Dostál 1935 (art. 2b, 31, 34a), Mughetum altherbosum silicicolum Dostál 1935 (art. 2b, 31, 34a), Mughetum (=Pinetum mughi) carpaticum Pawłowski 1956 p.p. (art. 2b, 31, 34a), Mughetum altherbosum calcicolum Zahradníková-Rošetzká 1957 (art. 2b, 31, 34a), Mughetum altherbosum silicicolum Zahradníková-Rošetzká 1957 (art. 31, 34a), Mughetum (silicicolum) herbosum Domin 1923 (nom. nud.), Pinetum mughi carpaticum Pawłowski 1928 (nom. nud.), Mughetum calcicolum Domin 1929 (nom. nud.).

Syntaxonomical synonyms: Athyrio-Pinetum mughi tatricum Hadač 1956 (art. 34a), Athyrio distentifolii-Pinetum mugo (Hadač 1956) Hadač in Mucina et Maglocký 1985 (art. 2b).

Phantom name: Athyrio-Pinetum mughi tatricum Sillinger 1933, Hadač 1956 (in Unar et al. 1984, 1985), AthyrioPinetum mugi (Sillinger 1933) Hadač 1956 (in Černušáková 1992).

Diagnostic taxa: Pinus mugo (dom.), Aconitum firmum (dif.), Adenostyles alliariae (dif.), Alchemilla sp. div. (dif.), Chaerophyllum hirsutum (dif.), Chrysosplenium alternifolium (dif.), Cicerbita alpina (dif.), Cortusa matthioli (dif.), Daphne mezereum (dif.), Doronicum austriacum (dif.), Epilobium montanum (dif.), Galeobdolon luteum s. 1. (dif.), Geranium sylvaticum (dif.), Geum rivale (dif.), Heracleum sphondylium (dif.), Hypericum maculatum (dif.), Leucanthemum rotundifolium (dif.), Lonicera nigra (dif.), Luzula sylvatica (dif.), Milium effusum (dif.), Myosotis sylvatica (dif.), Paris quadrifolia (dif.), Polygonatum verticillatum (dif.), Primula elatior (dif.), Ranunculus platanifolius (dif.), Ribes petraeum (dif.), Rosa pendulina (dif.), Rubus idaeus (dif.), Salix silesiaca (dif.), Senecio nemorensis agg. (dif.), Soldanella carpatica (dif.), Valeriana tripteris (dif.), Veratrum *lobelianum (dif.), Viola biflora (dif.), Calamagrostis villosa (const.), Homogyne alpina (const.), Oxalis acetosella (const.), Vaccinium myrtillus (const.), Dicranum scoparium (const.).

\section{DISCUSSION AND CONCLUSIONS}

In phytocoenological works of central European authors, the dwarf pine stands are commonly integrated to the alliance Pinion mugo and the class Vaccinio-Piceetea. The main reason for this is the presence of typical species of the mountain spruce forests (Svoboda 1939). Altogether, two or three types of Pinus mugo scrubs were distinguished there. At first, this division was attached to the differences in geology of the phytocoenoses occurrence (cf.: Szafer et al. 1927; Pawłowski et al. 1928), later it was based on the differences in carrying capacity of the soil or physiognomy (cf.: Sillinger 1933; Hadač 1956, 1985; Šoltésová 1974).

The great variety of opinions and views on the classification of dwarf pine stands in the Western Carpathians motivated us to perform a syntaxonomical revision, where besides classical phytocoenologic methods the numerical approach could be applied. The results of this revision can be summarized as follows:

1. According to Hadač (1956), we have assigned the $P i$ nus mugo scrubs from the Western Carpathians into three associations based of total physiognomy, stand conditions, and nutrient contents of the soil (Fig. 5). These groups of phytocoenoses are treated here as the following associations: Adenostylo alliariae-Pinetum mugo (Sillinger 1933) Šoltésová 1974, Cetrario islandicae-Pinetum mugo Hadač 1956 and Homogyno alpinae-Pinetum mugo (Sillinger 1933) nom. nov.

2. For floristically poor and monotonous communities on soils with a thick layer of undecomposed humus, we suggest the new name Homogyno alpinae-Pinetum mugo (Sillinger 1933) nom. nov. The names Vaccinio myrtilli-Pinetum mugo Hadač 1956 and Vaccinio myrtilli-Pinetum mugo (Sillinger 1933) Šoltésová 1974 are only the younger homonyms of the valid name of the association Vaccinio myrtilli-Pinetum montanae Morton 1927, which represents the acidophilous dwarf pine stands on calcareous bedrocks in the Alps. To treat the West Carpathian phytocoenoses as identical to this Alpine association was found to be erroneous. Besides the numerous phytogeographically important species occurring in only one of these two mountain ranges [Alps: Alnus alnobetula, Gentiana panonnica, Rhododendron ferrugineum, Rhododendron hirsutum, Rhododendron xintermedium, Veratrum album, Trolius europaeus etc.; (Western) Carpathians: Salix silesiaca, Soldanella carpatica, Veratrum *lobelianum etc.], the structure and floristic composition of respective stands is different; furthermore, the Western Carpathians stands are poorer floristically. The occurrence of plant species typical for tall-herb communities of the class Mulgedio-Aconitetea (Adenostyles alliariae, Cicerbita alpina, Geranium sylvaticum, Polygonatum verticillatum, Saxifraga rotundifolia, etc.) in the stands of the Alps-related association already indicates the different interpretation of the communities incorporated under these names (the Vaccinio myrtilli-Pinetum mugo and the Vaccinio myrtilli-Pinetum montanae), in different mountain systems (cf.: Morton 1927; Wallnöfer 1993a).

3. The most oligotrophic stands with dominating Sphagnum species in an underbrush, have been evaluated as a component of the association Homogyno alpinae-Pinetum mugo. The total physiognomy and floristic composition of these stands is more similar to this association, than to the association Cetrario-Pinetum mugo (syn. V.-P. cladonietosum rangiferino-gracilis), where they were classified to by Šoltésová (1974). These stands represent a connecting link between both associations.

The differences between the dwarf pine stands of the subalpine belt and the relic dwarf pine stands on raised bogs in the montane belt has already been pointed by Mia- 
dok (1976) in his description of the association Sphagnomughetum dealpinum. The question of their final classification and the comparison with relevant communities from the class Oxycocco-Sphagnetea will be discussed in our further paper (Šibík, in prep.).

4. The distinguishing of the association Athyrio distentifolii-Pinetum mugo (Hadač 1956) Hadač in Mucina et Maglocký 1985 (described from the granite part of the Vysoké Tatry Mts) appeared to be baseless, because the results of the numerical classification and ordination revealed its identity with the association Adenostylo-Pinetum mugo (described from the calcareous part of the Nízke Tatry Mts). The occurrence of the taxa typical for flowering species rich communities of tall broad-leaved herbs and ferns optimally developed at moist habitats (see Table 2) highlight that available nutrients and moisture in the soils are more important that geological bedrock.

5. Taking into account the obtained knowledge, the limited vertical distribution (the subalpine belt) of studied phytocoenoses, the similar physiognomy, and mutual close syngenetic relationships between individual dwarf pine associations, we confirm their current classification within the one alliance, the Pinion mugo. The distinguishing of associations to different alliances (cf. Hadač 1956; Jirásek 1996; Husová et al. 2002) seems to be pointless and unnecessary. To this fact pointed out already Gerdol and Piccoli (1982), who noticed that the floristic composition between Pinus mugo scrubs associations is not so sharp to justify their attribution to two different alliances and/or classes.

The incorporation of the alliance Pinion mugo to the order Piceetalia excelsae and to the class Vaccinio-Piceetea does not seem to be suitable, despite the similar floristic composition with the communities from the mountain spruce forests. The Pinus mugo scrubs create a distinctive vegetation belt above the timberline, in a zone, where the forest cannot naturally exist. Moreover, the physiognomy of stands is very different. The pure floristic approach clearly reaches the limits and consideration of physiognomic and habitat characteristics are indispensable (Theurillat et al. 1995). This was already, though indirectly, noted by the Polish authors Pawłowski et al. (1928), who have included the alliance Pinion mugo, only temporarily, however, to the order Piceetalia excelsae. They wrote: "Die Einreihung dieses Verbandes zur Ordnung der Piceetalia is bloß provisorisch. Vielleicht wird man das Pinetum mughi zusammen mit dem Alnetum viridis sowie anderen verwandten Strauchgesellschaften zu einer besonderen Vegetationsordnung zusammenfassen müssen". About fifty years later, Boşcaiu (1971) included this alliance into the new order Junipero-Pinetalia mugi Boşcaiu 1971. In the 1990s, Theurillat et al. $(1994,1995)$ described the new class Roso pendulinae-Pinetea mugo, which includes all mountain dwarf pine stands both on basic and acid substrata and they emended the content of the order Junipero-Pinetalia mugi. This system is actually accepted by us.

A recent study about Austrian forests confirmed that based on purely floristic similarity, the numerical method (TWINSPAN) classified the Pinus mugo scrubs within the communities dominated by Alnus alnobetula, Larix deci$d u a$, and Pinus cembra and together with the adjoining $P i$ cea forests. There are strong floristically connections between these vegetation types, but their physiognomic, biogeographic and ecological position is quite different. In so- me cases, the consideration of physiognomy and ecology is indispensable if we try to generate a system of global acceptance (Grabherr et al. 2003).

\section{ACKNOWLEDGEMENTS}

The authors are grateful to Dana Bernátová, Iveta Gažiová, Anna Guttová, Ivan Jarolímek, Peter Kučera, Patrik Mráz, Giuseppe Oriolo, Ján Ripka, Ivana Šibíková, Iveta Škodová, Jean-Paul Theurillat and Karol Ujházy for their valuable comments and suggestions on this paper, as well as for providing publications difficult to access. The processing of data with Canoco program was possible with co-operation of DAPHNE - Center for Applied Ecology. Our thanks go also to Radmila Bělohlávková and Ladislav Mucina for providing their unpublished phytocoenological relevés, and the anonymous reviewer for valuable suggestions on the manuscript. For English improvement we are indebted to Ivan Jarolímek, Daniela Michálková, Natália Yehorová and Ivana Šibíková. This work was supported by the grant agency VEGA, grant no. 4041.

\section{LITERATURE CITED}

AICHINGER E. 1933. Vegetationskunde der Karawanken. Gustav Fischer Verlag Jena, 329 pp.

ALTMANNOVÁ M. 1983. Subalpínska a alpínska vegetácia Nízkych Tatier a jej hodnotenie pre potreby LANDEP. Ph. D. Thesis. Institute of Botany, Slovak Academy of Sciences, Bratislava. (in Slovak)

BARKMAN J.J., DOING H., SEGAL S. 1964. Kritische Bemerkungen und Vorschläge zur quantitativen Vegetationsanalyse. Acta Bot. Neerl. 13: 394-419.

BĚLOHLÁVKOVÁ R. 1980. Rostlinná společenstva alpínského stupně Kriváňské Malé Fatry. Ph. D. Thesis. Faculty of Natural Sciences, Charles University, Prague. (in Czech)

BOŞCAIU N. 1971. Flora şi vegetaţia munţilor Ţarcu, Godeanu şi Cernei. Editura Academiei Republicii Socialiste România, Bucureşti, 494 pp. (in Rumanian)

BRAUN-BLANQUET J. 1964. Pflanzensoziologie. Grundzüge der Vegetationskunde. 3. Aufl. Springer Verlag, Wien, 865 pp.

BRAUN-BLANQUET J., SISSINGH G., VLIEGER J. 1939. Klasse der Vaccinio-Piceetea. Prodr. Groupements Vég. 6. Comité Int. Prodr. Phytosociol., Montpellier.

ČERNUŠÁKOVÁ D. 1992. Dwarf pine stands from Osobitá. Biologia 47/4: 341-349.

DOMIN K. 1923. Problémy a metody rostlinné sociologie. Publikace Ministerstva Zemědělství 39: 1-375. (in Czech)

DOMIN K. 1929. Příspěvek k poznání vegetačních poměrů a květeny Malého Havranu v Bielských Tatrách. Spisy Přír. Fak. Karlovy Univ. 1929/101: 3-18. (in Czech)

DÚBRAVCOVÁ Z., HAJDÚK J. 1986. Príspevok k výskumu vegetácie subalpínskeho stupňa Sivého vrchu v Západných Tatrách. Zborn. Slov. Nár. Múz., Prír. Vedy 32: 33-54. (in Slovak)

DÚBRAVCOVÁ Z. 1974. Subalpínska a alpínskeho vegetácia Kamenistej doliny (Západné Tatry). M.Sc. Thesis. Faculty of Natural Sciences, Comenius University, Bratislava. (in Slovak)

DÚBRAVCOVÁ Z., JAROLÍMEK I., KLIMENT J., PETRÍK A., ŠIBÍK J., VALACHOVIČ M. 2005. Alpine heaths in the Western Carpathians - a new approach to the classification. Ann. Bot. (in press.).

ELLENBERG H., WEBER H.E., DÜLL R., WIRTH W., WERNER W., PAULIßEN D. 1992. Zeigerwerte von Pflanzen in Mitteleuropa. Ed. 2. Scr. Geobot. 18: 1-258. 
GERDOL R., PICCOLI F. 1982. A phytosociological numerical study of the vegetation above the timberline on Monte Baldo (N-Italy). Phytocoenologia 10/4: 487-527.

GRABHERR G., REITER K., WILLNER W. 2003. Towards objectivity in vegetation classification: the example of the $\mathrm{Au}-$ strian forests. Plant Ecology 169: 21-34.

HADAČ E. 1956. Rostlinná společenstva Temnosmrečinové doliny ve Vysokých Tatrách. Biol. Práce Slov. Akad. Vied 2/1: 178. (in Czech)

HADAČ E. 1985. Pinion mugo Pawłowski et al. 1928. In: Mucina L., Maglocký Š. (eds), A list of vegetation units of Slovakia. Docum. Phytosociol. N. S. 9: 218.

HADAČ E., BŘEZINA P., JEŽEK V., KUBIČKA J., HADAČOVÁ V., VONDRÁČEK M. et al. 1969. Die Pflanzengesellschaften des Tales "Dolina Siedmich prameňov" in der Belauer Tatra. Vegetácia ČSSR, Veda, B2: 5-343.

HADAČ E., JEŽEK V., BŘEZINA P. 1969. Rostlinná společenstva Trojrohého plesa ve Vysokých Tatrách. Zborn. Pr. Tatran. Nár. Parku 11: 481-494. (in Czech)

HORÁK J. 1971. Westliche Tatra - Geobiozönosen der oberen Wald- und Krummholzgrenze. Acta Sc. Nat. Brno 5/5: 1-47.

HRABOVCOVÁ J. 1976. Vegetácia subalpínskeho a alpínskeho stupňa Jamnickej doliny (Západné Tatry). R.N.Dr. Thesis. Faculty of Natural Sciences, Comenius University, Bratislava. (in Slovak)

HUSOVÁ M., JIRÁSEK J., MORAVEC J. 2002. Přehled vegetace České republiky. Vegetation Survey of the Czech Republic. 3. Jehličnaté lesy. Coniferous forests. Academia, Praha, 128 pp. (in Czech)

JAROLÍMEK I., SCHLOSSER G. 1997. FYTOPACK - a system of programs to process phytocoenological tables. Biologia 52: 53-59.

JAROLÍMEK I., KLIMENT J., VALACHOVIČ M. 2002. The syntaxonomical revision of the riparian plant communities dominated by Petasites hybridus in Slovakia. Biologia 57/4: 471-492 .

JIRÁSEK J. 1996. Společenstva kosodřeviny (Pinus mugo) v České republice. Preslia 68: 1-12. (in Czech)

KLIKA J. 1926. Poznámky ke geobotanickému výzkumu Velké Fatry. Sborn. Přír. Společn. v Moravské Ostravě 3/1924-1925: 38-85. (in Czech)

KLIKA J. 1927. Příspěvek ke geobotanickému výzkumu Velké Fatry. 1. O lesních společenstvech. Preslia 5/1927: 3-35. (in Czech)

KLIMENT J., JAROLÍMEK I., ŠIBÍK J., VALACHOVIČ M. 2004. Syntaxonomy and nomenclature of the communities of the orders Calamagrostietalia villosae and Adenostyletalia in Slovakia. Thaiszia-J. Bot. 14/2: 93-157.

KOMÁRKOVÁ V. 1964. Alpinská vegetace Roháčů. M.Sc. Thesis. Faculty of Natural Sciences, Charles University, Prague. (in Czech)

KRAJINA V. 1933. Die Pflanzengesellschaften des Mlynica-Tales in den Vysoké Tatry (Hohe Tatra). 2. Teil. Beih. Bot. Centralbl. 51: 1-224.

KREMLOVÁ R. 1974. Alpínska a subalpínska vegetácia Žiarskej doliny (Západné Tatry). M.Sc. Thesis. Faculty of Natural Sciences, Comenius University, Bratislava. (in Slovak)

LUPIN M., FAŠKO P., MELO M. ŠŤASTNÝ P., TOMLAIN J. 2002. 27. Klimatické oblasti (1:1 000 000). In: Miklós L. (ed.), Atlas krajiny Slovenskej republiky, Ministerstvo životného prostredia SR, Bratislava \& Slovenská agentúra životného prostredia, Banská Bystrica, 344 pp. (in Slovak)

MARHOLD K., HINDÁK F. (eds). 1998. Checklist of non-Vascular and Vascular Plants of Slovakia. Veda, Bratislava, 687 pp.

MIADOK D. 1976. Ist das Knieholz (Pinus mugo ssp. mughus (Scop.) Zenari) im Hrdzavá-Tal natürlichen Ursprungs? Acta Fac. Rer. Natur. Univ. Comen. Bot. 25: 127-131.

MIADOK D. 1995. Vegetácia ŠPR Ďumbier. Univerzita Komenského, Bratislava, 72 pp. (in Slovak)
MILOVÁ M. 1993. Nelesné rastlinné spoločenstvá Štátnej prírodnej rezervácie Š́tovská dolina. Zbor. Orav. Múz. 1993: 17-24. (in Slovak)

MORTON F. 1927. Beiträge zur Soziologie ostalpiner Wälder. Bot. Arch. 19: 361-379.

MRÁZ K. 1956. Smilkové pastviny Martinských holí jako vývojová stadia lesních společenstev. Biológia Bratislava 11/1: 3-11. (in Czech)

MUCINA L., MAGLOCKÝ Š. 1985. A list of vegetation units of Slovakia. Docum. Phytosociol. N. S. 9: 175-220.

PAWŁOWSKI B. 1928. Guide des excursions en Pologne. 1. partie. Guide de l'excursion botanique dans les months Tatras. Cinquičme excursion phytogéographique internationale 1928: 1-61.

PAWŁOWSKI B. 1956. Flora Tatr. Rośliny naczyniowe. 1. Państw. Wydawn. Nauk, Warszawa, 672 pp. (in Polish)

PAWŁOWSKI B., STECKI K. 1927. Die Pflanzenassoziationen des Tatra-Gebirges. IV. Teil: Die Pflanzenassoziationen des Miętusia-Tales und des Hauptmassivs der Czerwone Wierchy. Bull. Int. Acad. Polon. Sci., Cl. Sci. Math., Ser. B, Sci. Nath., suppl. 2: 79-121.

PAWŁOWSKI B., SOKOLOWSKI M., WALLISCH K. 1928. Die Pflanzenassoziationen des Tatra-Gebirges. Teil 7. Die Pflanzenassoziationen und die Flora des Morskie Oko Tales. Bull. Int. Acad. Polon. Sci. Lett. 2: 197-272.

PODANI J. 1993. SYN-TAX-pc. Computer Programs for Multivariate Data Analysis in Ecology and Systematics. Ver. 5.0. Scientia Publ., Budapest, 104 pp.

POLDINI L., ORIOLO G., FRANCESCATO C. 2004. Mountain pine scrubs and heaths with Ericaceae in the south-eastern Alps. Plant Biosystems 138/1: 53-85.

RANDUŠKA D., VOREL J., PLÍVA K. 1986. Fytocenológia a lesnícka typológia. Príroda, Bratislava. 339 pp. (in Slovak)

ROUSSAKOVA V. 2000. Végétation alpine et sous alpine supérieure de la Montagne de Rila (Bulgarie). Braun-Blanquetia 25: 1-132.

SAMEK V., JANČǍ̌ÍK V., KRIESL A., MATERNA J. 1957. Lesní společenstva severního úbočí Vysokých Tater. (Část I. Javorová dolina). Lesn. Čas. 3/1: 3-38. (in Czech)

SILLINGER P. 1933. Monografická studie o vegetaci Nízkých Tater. Orbis, Praha, 339 pp. (in Czech)

SVOBODA P. 1939. Lesy Liptovských Tater. Studie o dřevinách a lesních společenstvech se zvláštním zřetelem k vlivům antropozooickým. Opera Bot. Čech. 1: 1-164. (in Czech)

SZAFER W., PAWŁOWSKI B., KULCZYŃSKI S. 1923. Die Pflanzenassoziationen des Tatra-Gebirges. I. Teil: Die Pflanzenassoziationen des Chochołowska-Tales. Bull. Int. Acad. Polon. Sci., Cl. Sci. Math., Ser. B, Sci. Nath., suppl. pp. 1-66.

SZAFER W., PAWŁOWSKI B., KULCZYŃSKI S. 1927. Die Pflanzenassoziationen des Tatra-Gebirges. III. Teil: Die Pflanzenassoziationen des Kościeliska-Tales. Bull. Int. Acad. Polon. Sci., Cl. Sci. Math., Ser. B, Sci. Nath., suppl. pp. 13-78.

ŠIBÍK J. 2003. Nelesné spoločenstvá subalpínskeho stupňa Krivánskej Malej Fatry. M.Sc. Thesis, Faculty of Natural Sciences, Comenius University, Bratislava. (in Slovak)

ŠIBÍK J., PETRÍK A., KLIMENT J. 2004. Syntaxonomical revision of plant communities with Carex firma and Dryas octopetala (alliance Caricion firmae) in the Western Carpathians. Polish Bot. J. 49/2: 181-202.

ŠMARDA J., LAZEBNÍČEK J., MATOUŠOVÁ L., NETOPIL R., PACLOVÁ L., PIKULA J. 1971. K ekologii rostlinných společenstev Doliny Sedmi pramenů v Belanských Tatrách. Práce Štúd. Českoslov. Ochr. Prír. 3/4: 1-204. (in Czech)

ŠOLTÉS R. 1976. Phytozönotische Analyse des Verbandes Vaccinio-Piceion Br.-B1. 1938 in den Westkarpaten. Acta Fac. Rer. Natur. Univ. Comen. Bot. 24: 139-167.

ŠOLTÉS R., HÁJEK M., VALACHOVIČ M. 2001. OxycoccoSphagnetea. In: Valachovič M. (ed.), Rastlinné spoločenstvá Slovenska. 3. Vegetácia mokradí. Veda, Bratislava, pp. 275 296. (in Slovak) 
ŠOLTÉSOVÁ A. 1974. Bestände des Knieholzes Pinus *mughus (Scop.) Zenari in den Westkarpaten (Phytozönotische Analyse und Vorschlag zur Einteilung von Knieholzbestände in den Westkarpaten). Acta Fac. Rer. Natur. Univ. Comen. Bot. 23: 79-104.

ŠOMŠÁK L. 1980. Lesné spoločenstvá Babej hory a Pilska. The annual report. Faculty of Natural Sciences, Comenius University, Bratislava. (in Slovak)

ŠOMŠÁK L. et al. 1981. Vplyv zošlapovania na vegetáciu okolia Skalnatého plesa a Hrebienka vo Vysokých Tatrách. Zborn. Pr. Tatran. Nár. Parku 22: 145-292. (in Slovak)

ter BRAAK C.J.F., ŠMILAUER P. 2002. CANOCO reference manual and CanoDraw for Windows user's guide. Software for canonical community ordination (version 4.5). Biometris, Wageningen \& České Budějovice, 500 pp.

THEURILLAT J.-P., AESCHIMANN D., KUPFER P., SPICHIGER R. 1994. Habitats et regions naturelles des Alpes. Colloq. Phytosoc. 22/1993: 16-30.

THEURILLAT J.-P., AESCHIMANN D., KUPFER P., SPICHIGER R. 1995. The higher vegetation units of the Alps. Colloq. Phytosoc. 23/1994: 189-239.

TICHÝ L. 2002. JUICE, software for vegetation classification. J. Veg. Sci. 13: 451-453.

UNAR J., UNAROVÁ M., ŠMARDA J. 1984. Vegetační poměry Tomanovy doliny a Žlebu spod Diery v Západních Tatrách. 1. Fytocenologické tabulky. Folia Fac. Sci. Nat. Univ. Purkynianae Brun., Ser. Biol. 25/10: 5-101. (in Czech)
UNAR J., UNAROVÁ M., ŠMARDA J. 1985. Vegetační poměry Tomanovy doliny a Žlebu spod Diery v Západních Tatrách. 2. Charakteristika př́rodních poměrů a rostlinných společenstev. Folia Fac. Sci. Nat. Univ. Purkynianae Brun., Ser. Biol. 26/14: 5-78. (in Czech)

van den MAAREL E. 1979. Transformation of cover-abundance values in phytosociology and its effect on community similarity. Vegetatio 39: 97-114.

VOLOŠČUK I. 1966. Príspevok k typologickému mapovaniu kosodreviny. Lesn. Čas. 39/8: 791-804. (in Slovak)

WALAS J. 1933. Roślinność Babiej Góry (Vegetation des Babia Góra-Gebietes in den Karpaten). Państwowa rada ochrony przyrody 2: 1-68. (in Polish)

WALLNÖFER S. 1993a. Erico-Pinetea. In: Mucina L., Grabherr G., Wallnöfer S. (eds), Die Pflanzengesellschaften Österreichs 3, pp. 244-282, Gustav Fischer Verlag Jena, Stuttgart, New York.

WALLNÖFER S. 1993b. Vaccinio-Piceetea. In: Mucina L., Grabherr G., Wallnöfer S. (eds), Die Pflanzengesellschaften Österreichs 3, pp. 283-337, Gustav Fischer Verlag Jena, Stuttgart, New York.

WEBER H.E., MORAVEC J., THEURILLAT J.-P. 2000. International Code of Phytosociological Nomenclature. Ed. 3. J. Veget. Sci. 11: 739-768.

ZAHRADNÍKOVÁ-ROŠETZKÁ K. 1957. Príspevok ku kvetene Demänovskej doliny. Biol. Práce Slov. Akad. Vied 3/4: 1-57. (in Slovak)

\section{APPENDIX}

Appendix 1 - Sources to the Table 2:

A: Cetrario islandicae-Pinetum mugo: 2 - Dúbravcová 1974: 88-92, rel. 127, 143, Západné Tatry Mts; 2 - Hadač 1956, Tab. 4, rel. 17, 116, Vysoké Tatry Mts; 7 - Horák 1971, Tab. 2, rel. 2628, 30, 32, 33, 37, Západné Tatry Mts; 4 - Komárková 1964, Tab. 11, rel. 1-4, Západné Tatry Mts; 1 - Kremlová 1974: 62, Západné Tatry Mts; 9 - Krajina 1933, Tab. 71, rel. 1-9, Vysoké Tatry Mts; 1 - Svoboda 1939: 106, Západné Tatry Mts; 2 - Šoltésová 1974, Tab. 2, rel. 37, 38, Západné Tatry Mts;

B: Homogyno alpinae-Pinetum mugo: 5 - Altmannová 1983, Tab. 23, rel. 1-5, Západné Tatry Mts; 10 - Bělohlávková 1980, Tab. 16, rel. 1-10, Krivánska Malá Fatra Mts; 1 - Bělohlávková 1980, Tab. 17, rel. 26, Krivánska Malá Fatra Mts; 6 - Černušáková 1992, Tab. 1, rel. 1-6, Západné Tatry Mts; 3 - Černušáková 1992, Tab. 2, rel. 1, 3, 5, Západné Tatry Mts; 16 Dúbravcová 1974: 88-92, rel. 120, 132-134, 141, 142, 153-155, 157-159, 163, 207-209, Západné Tatry Mts; 1 - Dúbravcová and Hajdúk 1986: 45, Západné Tatry Mts; 5 - Hadač 1956, Tab. 36, rel. 44-47, 115, Vysoké Tatry Mts; 3 - Hadač et al. 1969: 309310, rel. 137, 239, 252, Belianske Tatry Mts; 2 - Hadač, Ježek and Březina 1969: 490-491, rel. 11, 28, Vysoké Tatry Mts; 14 Horák 1971, Tab. 2, rel. 29, 31, 34-36, 38-43, 46-48, Západné Tatry Mts; 8 - Hrabovcová 1976, Tab. 14, rel. 1-8, Západné Tatry Mts; 4 - Komárková 1964, Tab. 7, rel. 1-4, Západné Tatry Mts; 10 - Krajina 1933, Tab. 69, rel. 1-10, Vysoké Tatry Mts; 8 Kremlová 1974: 26-30, rel. 46, 50, 168, 179, 182, 184, 185, 193, Západné Tatry Mts; 5 - Miadok 1995: 55-56, rel. 2-6, Nízke Tatry Mts; 1 - Milová 1993: 20, Krivánska Malá Fatra Mts; 1 Mráz 1956, Tab. 1, rel. 15, Lúčanská Malá Fatra Mts; 2 Pawłowski et al. 1928, Tab. 11, rel. 5, 6, Vysoké Tatry Mts; 4 Samek et al. 1957, Tab. 2, rel. 28-30, 36, Vysoké Tatry Mts; 4 Sillinger 1933: 123, rel. 1-4, Nízke Tatry Mts; 1 - Szafer et al. 1923, Tab. 6, rel. 153, Západné Tatry Mts; 13 - Szafer et al.
1927, Tab. 2, rel. 19-31, Západné and Vysoké Tatry Mts, Oravské Beskydy Mts; 1 - Šibík 2003: 68, Krivánska Malá Fatra Mts; 35 - Šoltésová 1974, Tab. 2, rel. 1-32, 34-36, Nízke, Západné, Vysoké and Belianske Tatry Mts, Krivánska Malá Fatra Mts; 2 Šomšák 1980: 28, rel. 1, 2, Oravské Beskydy Mts; 6 - Šomšák et al. 1981, Tab. 11, rel. 1-6, Vysoké Tatry Mts; 5 - Unar et al. 1984, Tab. 29, r. 1-5, Západné Tatry Mts; 5 - Unar et al. 1984, Tab. 38, rel. 1-5, Západné Tatry Mts; 10 - Unar et al. 1984, Tab. 39, rel. 1-10, Západné Tatry Mts; 4 - Vološčuk 1966, Tab. 1, rel. 12, 19, 20, 40, Západné Tatry Mts; 9 - Walas 1933, Tab. 15, rel. 6-8, 11-16, Oravské Beskydy Mts; 1 - Mucina ined., Nízke Tatry Mts;

C: Adenostylo alliariae-Pinetum mugo: 25 - Bělohlávková 1980, Tab. 17, rel. 1-25, Krivánska Malá Fatra Mts; 2 - Černušáková 1992, Tab. 2, rel. 2, 4, Západné Tatry Mts; 2 - Hadač 1956, Tab. 21, rel. 126, 127, Vysoké Tatry Mts; 2 - Hadač et al. 1969: 309-310, rel. 27, 122, Belianske Tatry Mts; 1 - Horák 1971, Tab. 2, rel. 44, Západné Tatry Mts; 3 - Klika 1926: 62-63, rel. 1-3, Velká Fatra Mts; 1 - Klika 1927: 31, Velká Fatra Mts; 1 - Komárková 1964: 96, Západné Tatry Mts; 5 - Krajina 1933, Tab. 62, rel. 3-7, Vysoké Tatry Mts; 1 - Miadok 1995: 54, Nízke Tatry Mts; 1 - Miadok 1995: 55-56, rel. 1, Nízke Tatry Mts; 4 Pawłowski et al. 1928, Tab. 11, rel. 1-4, Vysoké Tatry Mts; 6 Sillinger 1933: 120-121, rel. 1-6, Nízke Tatry Mts; 19 - Szafer et al. 1927, Tab. 2, rel. 1-18, 32, Západné and Vysoké Tatry Mts, Oravské Beskydy Mts; 5 - Šmarda et al. 1971, Tab. 11, rel. 122124, 126, 127, Belianske Tatry Mts; 1 - Šoltésová 1974, Tab. 2, rel. 33, Velká Fatra Mts; 20 - Šoltésová 1974, Tab. 3, rel. 1-20, Nízke, Západné, Vysoké and Belianske Tatry Mts, Krivánska Malá Fatra Mts, Velká Fatra Mts and Chočské vrchy Mts; 6 Unar et al. 1984, Tab. 28, rel. 1-6, Západné Tatry Mts; 2 - Walas 1933, Tab. 15, rel. 1, 2, Oravské Beskydy Mts; 1 -ZahradníkováRošetzká 1957: 14, Nízke Tatry Mts. 